Rev. Int. Contam. Ambie. 35 (1) 81-100, 2019

DOI: 10.20937/RICA.2019.35.01.06

\title{
IDENTIFICACIÓN DE LAS ZONAS CONTAMINADAS CON METALES PESADOS EN EL POLVO URBANO DE LA CIUDAD DE MÉXICO
}

\author{
Carmen DELGADO ${ }^{1}$, Francisco BAUTISTA ${ }^{2 *}$, Avto GOGICHAISHVILI ${ }^{1}$, José Luis CORTÉS ${ }^{2}$, \\ Patricia QUINTANA ${ }^{3}$, Daniel AGUILAR ${ }^{3}$ y Rubén CEJUDO ${ }^{1}$
}

${ }^{1}$ Laboratorio Universitario de Geofísica Ambiental, Instituto de Geofísica, Universidad Nacional Autónoma de México, Unidad Michoacán, Antigua Carretera a Pátzcuaro 8701, Col. Ex-Hacienda de San José de la Huerta, 58190 Morelia, Michoacán, México

${ }^{2}$ Laboratorio Universitario de Geofísica Ambiental, Centro de Investigaciones en Geografía Ambiental, Universidad Nacional Autónoma de México, Campus Morelia, Antigua Carretera a Pátzcuaro 8701, Col. Ex-Hacienda de San José de la Huerta, 58190 Morelia, Michoacán, México

${ }^{3}$ Departamento de Física Aplicada, Centro de Investigación y Estudios Avanzados del IPN Unidad Mérida, A.P. 73 Cordemex, 97310,Mérida, Yucatán, México

*Autor para correspondencia; leptosol@ciga.unam.mx

(Recibido mayo 2017; aceptado mayo 2018)

Palabras clave: contaminación, kriging indicador, norma oficial mexicana, plomo, vanadio

\section{RESUMEN}

Siete millones de personas mueren al año por cáncer relacionado con la contaminación ambiental. El polvo urbano (PU) contiene partículas $<10 \mu \mathrm{m}$ que afectan la salud humana. En la Ciudad de México (CDMX) existe sólo un estudio de PU. El objetivo de este estudio fue identificar las zonas de la CDMX con mayor probabilidad de contaminación por metales pesados (MP) en el PU. Se recolectaron 89 muestras de PU y se midió la concentración de MP por fluorescencia de rayos X. El análisis espacial se realizó mediante interpolación con kriging indicador. El porcentaje de la superficie con mayor probabilidad de que los MP superen los límites máximos permitidos (LMP) fueron: Cr (LMP $280 \mathrm{mg} / \mathrm{kg}$ ), $0.55 \%$, suroeste; Cr (LMP $64 \mathrm{mg} / \mathrm{kg}$ ), 89 \%, toda la $\mathrm{CDMX}$ y la mayoría de las áreas conurbadas (AC); $\mathrm{Cu}, 17 \%, 11$ delegaciones y $\mathrm{AC}$ del norte; Ni, $13 \%$, centro y suroeste; $\mathrm{Pb}$ (LMP $140 \mathrm{mg} / \mathrm{kg}$ ), $17.5 \%$, delegaciones céntricas y AC del noroeste; $\mathrm{Pb}$ (LMP $400 \mathrm{mg} / \mathrm{kg}$ ), $2.2 \%$, delegaciones céntricas y AC del noroeste; V, $26 \%$, delegaciones céntricas y del sur, así como AC del noreste; $\mathrm{Zn}, 21 \%$, delegaciones céntricas y AC del norte. Los elementos con mayor número de sitios y superficie contaminada son: $\mathrm{Cr}, \mathrm{Zn}, \mathrm{Cu}$ y $\mathrm{Pb}$. Los sitios con mayor superficie de sobreposición de MP son aquellos con asociaciones entre $\mathrm{Cu}$ y $\mathrm{Zn}(15 \%)$, y $\mathrm{Cu}$ y $\mathrm{Pb}(13 \%)$. Las delegaciones Cuauhtémoc, Venustiano Carranza y Gustavo A. Madero son las más contaminadas con metales pesados.

Key words: pollution, kriging indicator, Official Mexican Standard, lead, vanadium 


\begin{abstract}
Seven million people die per year of cancer related to environmental pollution. Urban dust (UD) contains particles smaller than $10 \mu \mathrm{m}$ that affect human health. In Mexico City (CDMX) only one study on UD has been conducted. The objective of this study was to identify the areas most likely to be contaminated by heavy metals (HM) contained in the UD of CDMX. Eighty nine UD samples were collected; the analysis of the HM concentration was made by X-ray fluorescence, and spatial analysis by interpolation with kriging indicator. The percentage of the area with the highest probability that HMs exceeded the maximum permitted limits (MPL) were: Cr (MPL $280 \mathrm{mg} / \mathrm{kg}$ ), $0.55 \%$, southwest of the CDMX; Cr (MPL $64 \mathrm{mg} / \mathrm{kg}$ ), $89 \%$, all CDMX and most of the conurbated areas $(\mathrm{CA}) ; \mathrm{Cu}, 17 \%, 11$ municipalities and the northern $\mathrm{CA}$; $\mathrm{Ni}, 13 \%$, center and southwest of the CDMX; Pb (MPL $140 \mathrm{mg} / \mathrm{kg}$ ), $17.5 \%$, centric municipalities and the northwest $\mathrm{CA} ; \mathrm{Pb}$ (MPL $400 \mathrm{mg} / \mathrm{kg}$ ), $2.2 \%$, centric municipalities and the northwest $\mathrm{CA} ; \mathrm{V}, 26 \%$, centric and southern municipalities and the northeast $\mathrm{CA} ; \mathrm{Zn}$, $21 \%$, centric municipalities and the northern CA. Elements with a greater number of sites and contaminated surface are: $\mathrm{Cr}, \mathrm{Zn}, \mathrm{Cu}$ and $\mathrm{Pb}$. The largest $\mathrm{HM}$ overlapping sites are those with $\mathrm{Cu}$ and $\mathrm{Zn}$ associations (15\%), and $\mathrm{Cu}$ and $\mathrm{Pb}$ associations $(13 \%)$. The municipalities Cuauhtémoc, Venustiano Carranza and Gustavo A. Madero are the most contaminated with heavy metals.
\end{abstract}

\section{INTRODUCCIÓN}

Recientemente, la Organización Mundial de la Salud declaró que alrededor de siete millones de personas por año mueren por cáncer y otras enfermedades relacionadas con la contaminación del aire (WHO 2014). La contaminación del aire, agua y suelos ha sido suficientemente estudiada y existen normas ambientales que la regulan, pero no así el polvo (García-Rico et al. 2016).

No existen normas oficiales que regulen la presencia de polvo en el ambiente, a pesar de que este contiene partículas menores a $10 \mu \mathrm{m}$, como las $\mathrm{PM}_{10}$, $\mathrm{PM}_{2.5}$ y $\mathrm{PM}_{0.1}$, que son fácilmente inhalables y de alto riesgo para la salud humana porque albergan metales pesados (MP) potencialmente tóxicos como $\mathrm{Pb}, \mathrm{Cu}$ y Zn (Jarup 2003, Luo et al. 2012, Sabath y Robles-Osorio 2012).

El polvo proviene de fuentes naturales y antrópicas; sin embargo, el polvo urbano (PU) está compuesto principalmente de partículas derivadas de procesos antrópicos (García-Rico et al. 2016) como la industria (Liu et al. 2014), transporte vehicular y marítimo (Zafra et al. 2011, Aguilar Reyes et al. 2013, Sánchez-Duque et al. 2015, Cortés et al. 2017) y, en menor medida, la erosión de los suelos (Luo et al. 2012, Chen et al. 2014).

Las partículas finas del PU son emitidas a la atmósfera. Después de un tiempo, dependiendo del las condiciones climáticas, se depositan sobre suelos de parques, avenidas, banquetas, techos de casas y edificios, así como en superficies del arbolado urbano (Bautista et al. 2011, Chen et al. 2014, Liu et al. 2014).

Hay varios estudios de la ciudad de México (CDMX) y zonas conurbadas del Estado de México sobre contaminación por MP en partículas aéreas (Vallejo et al. 2003, Molina et al. 2010, en suelos (Rodríguez-Salazar et al. 2011, Cejudo et al. 2015a, Ihl et al. 2015) y en plantas (Guzmán-Morales et al. 2011). Sin embargo, sólo se ha realizado un estudio sobre PU, en el cual se reportan sus propiedades magnéticas (Cejudo et al. 2015b).

Por las características geológicas, de relieve y dirección de los vientos, es probable que la mayor parte de la CDMX esté contaminada por los MP contenidos en el PU, ya que en esa urbe habitan 23 millones y medio de personas, hay 40000 pequeñas y medianas industrias de diversos giros y transitan cuatro millones de vehículos automotores (FIMEVIC 2012).

Por lo anterior, se requieren estudios espacialmente explícitos para identificar las zonas más contaminadas de la zona metropolitana de la CDMX. El método de interpolación con kriging indicador (KI) permite diseñar mapas con diferentes niveles de probabilidad de contaminación, ya que es un estimador lineal con mínimo error (Webster y Oliver 1990, Burrough y McDonnell 1998, Goovaerts 1999).

El método KI se utiliza cuando la distribución de la población no es gaussiana (Antunes y Alburqueque 2013, Juang et al. 2004, Ihl et al. 2015) y se tienen umbrales de un contaminante. En este caso, los umbrales fueron los límites máximos permitidos 
(LMP) de los MP en suelos de acuerdo con las normas ambientales mexicanas y canadienses, a falta de normatividad de PU.

El objetivo de este estudio fue la identificación de las zonas con mayor probabilidad de contaminación por metales pesados contenidos en los polvos urbanos de la CDMX y áreas conurbadas con la finalidad de que este tipo de estudios, espacialmente explícitos, permitan una mejor y más efectiva intervención o para la instrumentación de medidas de saneamiento ambiental.

\section{MATERIALES Y MÉTODOS}

Área de estudio, muestreo y tratamiento de la muestra

La Ciudad de México está construida sobre depósitos volcánicos en el sur y oeste de la ciudad, y lacustres en el centro y el este (Díaz-Rodríguez 2006). Se localiza a una altura de $2240 \mathrm{msnm}$, en una cuenca semirrodeada de volcanes. Los vientos predominantes son del noreste hacia el suroeste (Vallejo et al. 2003) (Fig. 1).

Se diseñó un muestreo sistemático en el área de estudio de $1600 \mathrm{~km}^{2}$ mediante una rejilla de $10 \times 10$. Cada celda tuvo un borde de $4 \mathrm{~km}$ y un área de $16 \mathrm{~km}^{2}$. Para los análisis espaciales se recomienda un tamaño de muestra de 30 a 100 sitios a fin de que la varianza de la estimación sea mínima (Webster y Oliver 1990, Cejudo et al. 2015a, b, Ihl et al. 2015).

Se recolectaron 89 muestras de PU en la estación seca (marzo-abril) en $1 \mathrm{~m}^{2}$ en las vialidades a un lado de las banquetas. Se retiraron las piedras, hojas y ramas, y con una brocha se acumuló el polvo en los cuatro extremos del cuadrado; se recogió con un utensilio de plástico y se depositó en bolsas de polietileno etiquetadas y georreferenciadas.

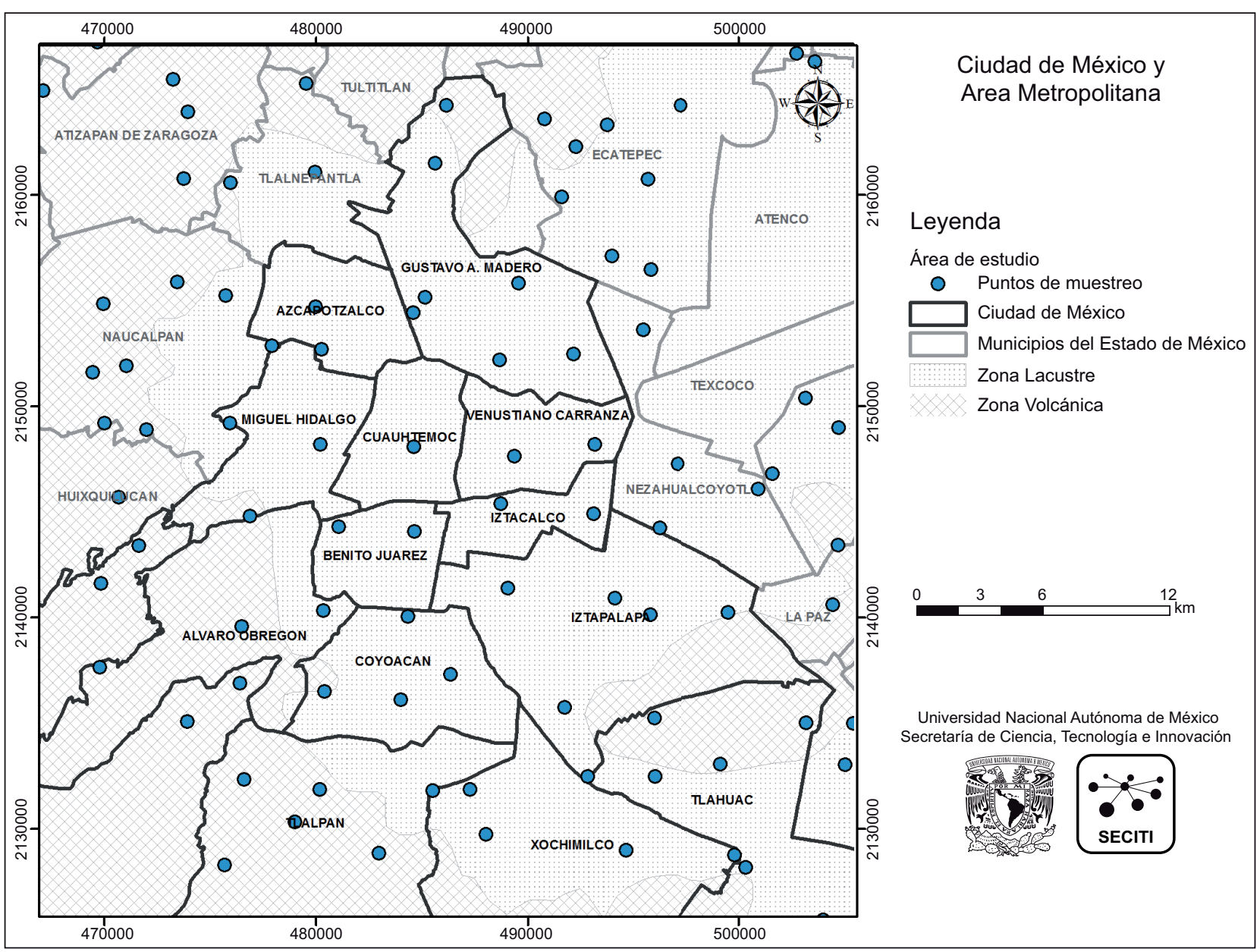

Fig 1. CDMX subdividida en delegaciones políticas y zonas conurbadas 
Dependiendo de las condiciones del sitio, en algunas ocasiones los puntos de muestreo definidos previamente se cambiaron a otros sitios, tratando de mantenerlos lo más cerca posible del punto original, dentro de la celda correspondiente.

Las muestras de PU se secaron durante dos semanas bajo la sombra a temperatura ambiente para evitar la oxidación de los minerales. Se molieron y tamizaron con malla de $2 \mathrm{~mm}$ y se volvieron a moler en un mortero de ágata, ya que a mayor homogeneidad de las muestras la precisión de las mediciones aumenta (Wei y Yang 2010, Bautista et al. 2011).

\section{Análisis químicos}

Las muestras de PU se comprimieron en un dado de $1 \mathrm{~cm}$ de diámetro sin ningún tratamiento químico o agentes aglutinantes, para obtener pastillas que se colocaron en un portamuestras y sellaron con película de poliéster (mylar).

Se cuantificaron las concentraciones totales de $\mathrm{Cr}$, $\mathrm{Cu}, \mathrm{Ni}, \mathrm{Pb}, \mathrm{V}$ y Zn mediante la técnica de fluorescencia de rayos $\mathrm{X}$ de energía dispersa (ED-XRF) en el Centro de Investigación y de Estudios Avanzados de Mérida, Yucatán. La técnica es cuantitativa, rápida, no destructiva ni contaminante (Lozano y Bernal 2005, Beckhoff et al. 2006).

La técnica ED-FRX es cuantitativa, ya que utiliza estándares para generar una curva de calibración de intensidad contra concentración, para después comparar las intensidades de las muestras con las de los estándares (Khodeir et al. 2012, Paltridge et al. 2012, Chen et al. 2014, Ihl et al. 2015, Cortés et al. 2017).

Se utilizaron estándares de referencia certificados internacionalmente para rocas y suelos. Como control de medición se consideró el estándar con clave de identificación IGLsy-1, el cual corresponde a una sienita de nefelina con alto contenido de $\mathrm{Al}$ y $\mathrm{Si}$ (Lozano y Bernal 2005, Beckhoff et al. 2006).

El espectrómetro ED-XRF es marca Jordan Valley EX-6600 equipado con un detector $\mathrm{Si}$ (Li), área activa de $20 \mathrm{~mm}^{2}$ y resolución de $140 \mathrm{eV}$ a $5.9 \mathrm{keV}$. Opera a un máximo de $54 \mathrm{kV}$ y $4800 \mu \mathrm{A}$, en condiciones de vacío atmosférico, utilizando una tarjeta secundaria intercambiable y tiempo de medición de 300 s. Cada medición se repitió cinco veces.

\section{Análisis de datos}

Se realizó la estadística descriptiva de los MP en el PU para conocer la distribución de la muestra. El análisis espacial se realizó mdeiante interpolación con KI, para la cual se requieren valores umbrales que pueden ser los LMP de los MP determinados por normas ambientales. Se utilizó el programa
Geostatistics for the Environmental Sciences (GS+) (Robertson 2008).

Debido a que no existe regulación ambiental para los PU, tanto en México como en el resto del mundo, en esta investigación se usaron como valores umbrales los LMP para suelo urbano establecidos por la NOM 147 SEMARNAT/SSA1-2004 (SEMARNAT 2007), así como las normas estadounidense (USEPA 2004) y canadiense (CCME 2007).

Los umbrales fueron los siguientes. Norma canadiense: $\mathrm{Cr}, 64 \mathrm{mg} / \mathrm{kg}$; $\mathrm{Cu}, 63 \mathrm{mg} / \mathrm{kg}$; Ni, $50 \mathrm{mg} / \mathrm{kg}$; $\mathrm{Pb}, 140 \mathrm{mg} / \mathrm{kg}, \mathrm{y} \mathrm{Zn}, 200 \mathrm{mg} / \mathrm{kg}$ ). Normas mexicana y estadounidense: $\mathrm{Pb}, 400 \mathrm{mg} / \mathrm{kg}, \mathrm{y} \mathrm{V}, 78 \mathrm{mg} / \mathrm{kg}$. Norma mexicana para el Cr: $280 \mathrm{mg} / \mathrm{kg}$.

A diferencia de kriging ordinario, el método de interpolación con KI no intenta conseguir un mapa de isolíneas de concentración, sino un mapa con las diferentes zonas de probabilidad de rebasar el valor umbral.

El KI hace una transformación binaria de los datos (0-1) para que el estimador sea menos sensible a los datos extremos. El valor 1 indica la máxima probabilidad de que la concentración del MP sea igual o mayor que el LMP; los valores menores a 1 indican algún grado de probabilidad de rebasarlo. Se calcula de la siguiente manera (Goovaerts 1999):

$I\left(x_{i} ; z_{k}\right)=\left\{\begin{array}{l}1, \operatorname{cuando} Z\left(x_{i}\right) \leq Z_{k} \\ 0, \text { cuando } Z\left(x_{i}\right) \geq Z_{k}\end{array} \quad \mathrm{k}=1, \ldots, \mathrm{K}\right.$

donde $I\left(x_{i} ; z_{k}\right)$ es la variable indicadora, $z\left(x_{i}\right)$ es la variable medida y $z_{k}$ es el umbral deseado.

El semivariograma indicador experimental se calcula de la siguiente manera:

$\lambda_{I}{ }^{*}(h)=\frac{1}{2 N(h)} \sum_{i=1}^{N(h)}\left[I\left(x_{i} ; z_{k}\right)-I\left(x_{i}+h ; z_{k}\right)\right]^{2}$

donde $N(h)$ es el número de pares indicadores que transforman a $I\left(x_{i} ; z_{k}\right)$ e $I\left(x_{i}+h ; z_{k}\right)$ separados por una distancia $h$.

La función de distribución acumulativa condicional en el sitio $x_{0}$ es:

$F\left(\frac{x_{0} ; z_{k}}{N}\right)=I^{*}\left(x_{0} ; z_{k}\right)=\sum_{i=1}^{N} \lambda_{i} I\left(x_{i} ; z_{k}\right)$

donde $I^{*}\left(x_{0} ; z_{k}\right)$ es el nivel del indicador estimado en el sitio no medido y $\lambda_{i}$ es el peso asignado al valor del indicador conocido $I\left(x_{i} ; z_{k}\right)$.

En los mapas se señalaron las zonas con los valores en el intervalo de 0.8 a 1 para ser lo más precisos en la descripción de los sitios con la máxima contaminación, los cuales deben atenderse con más urgencia.

Los mapas se realizaron con el programa ArcGis 9.0 (ESRI 2004), proyección universal transversal 
de Mercator (UTM), zona 14, elipsoide y datum horizontal Sistema Geodésico Mundial (WGS84).

El álgebra de mapas de los sitios de mayor superficie de sobreposición de MP se realizó con las superficies que tienen mayor probabilidad de superar el umbral considerando todos los metales analizados.

\section{RESULTADOS Y DISCUSIÓN}

Los valores de las concentraciones de los elementos $\mathrm{Cr}, \mathrm{Cu}, \mathrm{Ni}, \mathrm{Pb}, \mathrm{y} \mathrm{Zn}$ en $\mathrm{PU}$ de la CDMX no tienen una distribución gaussiana o normal, ya que los coeficientes de asimetría y curtosis estandarizados no están en el intervalo de -2 a 2, por lo que podría considerarse que estos elementos tienen un origen antrópico (Guvenç et al. 2003) (Cuadro I).

Los valores de la mediana de las concentraciones de $\mathrm{Cr}, \mathrm{Cu}, \mathrm{Pb}, \mathrm{V}$ y $\mathrm{Zn}$ son mayores a los valores de fondo de la mediana de muestras de suelos de origen natural de la CDMX (Morton-Bermea et al. 2009), lo cual refuerza la consideración de un origen principalmente antrópico (Cuadro II).

Los valores de la concentración de Ni en el PU no tienen una distribución gaussiana pero son menores a los valores de fondo de suelos de origen natural de la CDMX, por lo que podría considerarse que tienen origen natural (Cuadro II).

En muestras de suelo de la CDMX se ha reportado que $\mathrm{Pb}, \mathrm{Cu}$ y $\mathrm{Zn}$ son de origen antrópico, derivado del tráfico vehicular y la industria. Por el contrario, el origen de V, Ni y Cr no está del todo claro (Rodríguez-Salazar et al. 2011, Ihl et al. 2015).

El V se encuentra en rocas volcánicas (Cuadro II) pero también puede ser liberado al ambiente como óxidos de vanadio por la combustión de petróleo y carbón (ATSDR 2010), así como emisiones de automóviles. El petróleo americano contiene más $\mathrm{V}$ y el
$91 \%$ del V liberado al ambiente proviene de fuentes antrópicas (Rodríguez-Mercado y Altamirano-Lozano 2006, Liu et al. 2014).

De acuerdo con las normas ambientales canadienses, el porcentaje de las muestras que rebasaron los LMP para el suelo urbano fueron: $\mathrm{Cr}, 98 \%$; Zn, $80 \%$; $\mathrm{Cu}, 72 \%$; Pb, $63 \%$, y Ni, 44 \%. Sin embargo, utilizando las normas mexicanas, los resultados fueron los siguientes: $\mathrm{Cr}, 1 \%$; $\mathrm{Pb}, 7$ \%, y V, $74 \%$ de las muestras (Cuadro II).

Según las normas mexicanas, sólo el $\mathrm{Cr}, \mathrm{Pb}, \mathrm{V}$ significarían problemas de contaminación en el 1 , 7 y $74 \%$ de las muestras, respectivamente. Por el contrario, de acuerdo con las normas canadienses el $\mathrm{Cr}, \mathrm{Cu}, \mathrm{Ni}, \mathrm{Pb}$ y $\mathrm{Zn}$ rebasan los LMP entre el 44 y $98 \%$ de las muestras.

\section{Análisis espacial}

Los modelos de los variogramas tuvieron valores de $\mathrm{r}^{2}$ mayores de 0.8 en el $\mathrm{Cu}, \mathrm{Pb}, \mathrm{y} \mathrm{Zn}$, lo cual indica que existe una alta correlación espacial donde los modelos explican más del $60 \%$ de la dependencia espacial, lo cual es consistente con lo reportado en suelos de la CDMX por Rodríguez-Salazar et al. (2011) e Ihl et al. (2015). Esto indica que la estructura espacial está altamente influida por la contaminación derivada de las actividades antrópicas (Cuadro III).

En el Cr, Ni y V, $r^{2}$ fue menor de 0.6, lo que significa que existe baja correlación espacial (Cuadro III). Esta estructura espacial parece no estar influida por la contaminación, sino por el intemperismo natural de la roca madre, como reportan Rodríguez-Salazar et al. (2011) e Ihl et al. (2015) para los suelos de la CDMX.

El método de interpolación con KI mediante la transformación binaria de los datos (0-1) permitió que el estimador fuera menos sensible a datos extremos. El valor 1 indicó la máxima probabilidad de que la

CUADRO I. ESTADÍSTICA DESCRIPTIVA DE LOS METALES PESADOS EN POLVOS URBANOS DE LA CIUDAD DE MÉXICO

\begin{tabular}{lrrrrrr}
\hline Metal & Media & $\begin{array}{c}\text { Desviación } \\
\text { estándar }\end{array}$ & Máximo & Mínimo & $\begin{array}{c}\text { Kurtosis } \\
\text { estandarizada }\end{array}$ & $\begin{array}{c}\text { Asimetría } \\
\text { estandarizada }\end{array}$ \\
\cline { 2 - 7 } & \multicolumn{7}{c}{ ( } & & & $(\mathrm{mg} / \mathrm{kg})$ & & \\
\hline $\mathrm{Cr}$ & 126 & 126 & 1258 & 40 & 148.03 & 32.6 \\
$\mathrm{Cu}$ & 97 & 61 & 340 & 10 & 5.41 & 5.6 \\
$\mathrm{Ni}$ & 51 & 28 & 151 & 0 & 4.02 & 3.65 \\
$\mathrm{~Pb}$ & 206 & 128 & 610 & 17 & 1.08 & 3.43 \\
$\mathrm{~V}$ & 88 & 15 & 119 & 48 & -0.07 & -1.19 \\
$\mathrm{Zn}$ & 321 & 150 & 935 & 114 & 6.25 & 5.80 \\
\hline
\end{tabular}


CUADRO II. LÍMITES MÁXIMOS PERMITIDOS EN SUELOS URBANOS POR DIFERENTES PAÍSES Y NÚMERO DE MUESTRAS DE POLVOS QUE REBASAN LAS NORMAS DE MÉXICO, EUA Y CANADÁ

\begin{tabular}{lcccccc}
\hline País & $\mathrm{Cr}$ & $\mathrm{Cu}$ & $\mathrm{Ni}$ & $\mathrm{Pb}$ & $\mathrm{V}$ & $\mathrm{Zn}$ \\
\hline \multicolumn{7}{c}{$\mathrm{LMP}(\mathrm{mg} / \mathrm{kg})$} \\
\hline México & 280 & $\mathrm{~N} / \mathrm{A}$ & 1600 & 400 & 78 & $\mathrm{~N} / \mathrm{A}$ \\
EUA & 210 & 3100 & 1600 & 400 & 78 & 23000 \\
Canadá & 64 & 63 & 50 & 140 & N/A & 200 \\
\hline
\end{tabular}

Número de muestras de polvos que rebasan el LMP

\begin{tabular}{lcccccc}
\hline México-EUA & 1 & 0 & 0 & 6 & 66 & 0 \\
Canadá & 87 & 64 & 39 & 56 & N/A & 71 \\
\hline
\end{tabular}

Valores de fondo de la mediana en muestras de suelos de la $\mathrm{CDMX}^{1}$

\begin{tabular}{cccccc}
\hline 105 & 32 & 56 & 19 & 87 & 76 \\
\hline \multicolumn{6}{c}{ Valores de fondo de la mediana de muestras de } \\
polvos de este estudio
\end{tabular}

${ }^{1}$ Morton-Bermea et al. 2009

LMP: límite máximo permitido; N/A: no aplica

CUADRO III. PARÁMETROS DE LOS VARIOGRAMAS DE METALES PESADOS EN POLVOS URBANOS DE LA CDMX

\begin{tabular}{llccccl}
\hline $\begin{array}{l}\text { Metal } \\
\mathrm{N}=89\end{array}$ & Modelo & $\mathrm{r}^{2}$ & $\begin{array}{r}\text { Estructura } \\
\text { espacial } \%\end{array}$ & Distancia & $\begin{array}{c}\text { Nugget } \\
\%\end{array}$ & $\mathrm{VC}$ \\
\hline $\mathrm{Cr}$ & Gaussiano & 0.43 & 99 & 4156 & 0.01 & 0.003 \\
$\mathrm{Cu}$ & Exponencial & 0.85 & 91 & 12420 & 9 & 0.18 \\
$\mathrm{Ni}$ & Esférico & 0 & 89 & 1250 & 10 & 0.03 \\
$\mathrm{~Pb}$ & Exponencial & 0.87 & 91 & 18300 & 9 & 0.42 \\
$\mathrm{~V}$ & Esférico & 0.42 & 99 & 2900 & 1 & 0.1 \\
$\mathrm{Zn}$ & Esférico & 0.8 & 60 & 15660 & 40 & 0.2 \\
\hline
\end{tabular}

concentración del MP fuera igual o mayor que el LMP, y los valores menores de 1 indicaron algún grado de probabilidad de rebasarlos.

\section{Cromo}

El área con mayor probabilidad de contaminación por $\mathrm{Cr}$, de acuerdo con el LMP de $280 \mathrm{mg} / \mathrm{kg}$ establecido por la NOM 147-SEMARNAT/SSA1-2004 (SEMARNAT 2007) se localiza al suroeste de la CDMX en la delegación Álvaro Obregón (Fig. 2). Abarca una superficie de 1495 ha y corresponde al
$17.5 \%$ de la superficie delegacional, al $1.09 \%$ de la CDMX y $0.55 \%$ de la superficie muestreada.

De acuerdo con el LMP de $280 \mathrm{mg} / \mathrm{kg}$ establecido por la normatividad mexicana, en el área conurbada (AC) con el Estado de México no existen zonas en que las concentraciones de $\mathrm{Cr}$ rebasen ese valor (Cuadro IV).

De acuerdo con la norma canadiense (CCME 2007), cuyo LMP es de $64 \mathrm{mg} / \mathrm{kg}$, el área con mayor probabilidad de contaminación por $\mathrm{Cr}$ en la CDMX abarca una superficie de 127075 ha y corresponde al 


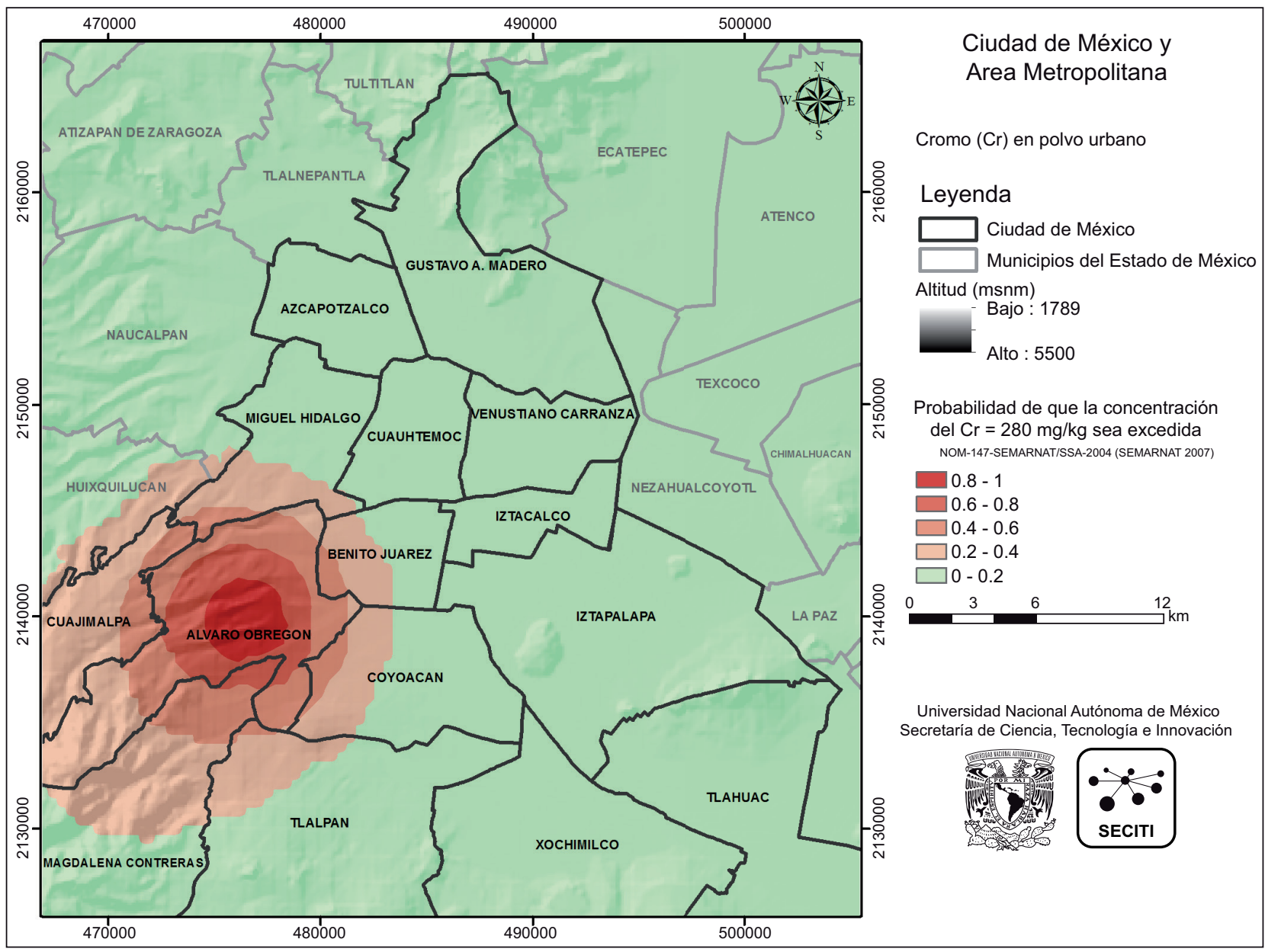

Fig 2. Áreas contaminadas con Cr en polvos urbanos de la CDMX con el LMP de $280 \mathrm{mg} / \mathrm{kg}$ establecido por las normas mexicanas (SEMARNAT 2007)

CUADRO IV. SUPERFICIES CONTAMINADAS POR MP EN POLVOS DE LA CIUDAD DE MÉXICO POR DELEGACIÓN Y ZONAS CONURBADAS CON EL ESTADO DE MÉXICO

\begin{tabular}{lrrrrrrrr}
\hline & \multicolumn{7}{c}{$\mathrm{MP} /$ límite máximo permitido $(\mathrm{mg} / \mathrm{kg})$} \\
\cline { 2 - 8 } Delegación/superficie (ha) & $\mathrm{Cr}$ & $\mathrm{Cr}$ & $\mathrm{Cu}$ & $\mathrm{Ni}$ & $\mathrm{Pb}$ & $\mathrm{Pb}$ & $\mathrm{V}$ & $\mathrm{Zn}$ \\
& 280 & 64 & 63 & 50 & 400 & 140 & 78 & 200 \\
\hline Cuauhtémoc (3252) & 0 & $\mathbf{3 2 5 2}$ & $\mathbf{3 2 5 2}$ & 859 & 915 & $\mathbf{3 2 5 2}$ & $\mathbf{3 2 5 2}$ & $\mathbf{3 2 5 2}$ \\
Azcapotzalco (3385) & 0 & $\mathbf{3 3 8 5}$ & $\mathbf{3 3 8 5}$ & 35 & 993 & $\mathbf{3 3 8 5}$ & 665 & $\mathbf{3 3 8 5}$ \\
Iztacalco (2303) & 0 & $\mathbf{2 3 0 2}$ & $\mathbf{2 3 0 2}$ & 0 & 82 & $\mathbf{2 2 2 0}$ & 30 & $\mathbf{2 3 0 2}$ \\
Venustiano Carranza (3355) & 0 & $\mathbf{3 3 5 5}$ & $\mathbf{3 3 5 5}$ & 726 & 0 & $\mathbf{3 2 9 2}$ & 545 & $\mathbf{3 3 5 5}$ \\
Miguel Hidalgo (4679) & 0 & $\mathbf{4 6 7 9}$ & $\mathbf{4 3 7 6}$ & 2 & 169 & $\mathbf{4 5 3 3}$ & 2706 & $\mathbf{4 2 4 6}$ \\
Benito Juárez (2675) & 0 & $\mathbf{2 6 7 5}$ & $\mathbf{2 6 7 5}$ & 2 & 525 & 1267 & 580 & $\mathbf{2 6 7 5}$ \\
Iztapalapa (11351) & 0 & $\mathbf{9 5 5 8}$ & $\mathbf{1 0 3 7 0}$ & 0 & 732 & 5245 & 4973 & $\mathbf{1 0 3 1 1}$ \\
Gustavo A. Madero (8720) & 0 & $\mathbf{8 7 2 0}$ & 1101 & 2524 & 0 & 3017 & 5004 & $\mathbf{6 9 6 9}$ \\
Magdalena Contreras (10765) & 0 & $\mathbf{1 0 7 6 5}$ & 0 & $\mathbf{1 0 7 6 5}$ & 0 & 0 & 4150 & 0 \\
Xochimilco (12714) & 0 & $\mathbf{1 2 7 1 4}$ & 0 & 0 & 0 & 0 & $\mathbf{1 2 7 1 4}$ & 1504 \\
Coyoacán (5373) & 0 & $\mathbf{5 3 7 3}$ & 3694 & 1004 & 0 & 0 & 1050 & 4905 \\
Tlalpan (32519) & 0 & $\mathbf{3 2 5 1 9}$ & 0 & 17382 & 0 & 0 & 23378 & 2819 \\
Álvaro Obregón (8527) & 1495 & $\mathbf{8 5 2 7}$ & 1983 & 2688 & 0 & 1095 & 481 & 1589 \\
Cuajimalpa (17323) & 0 & $\mathbf{1 7 3 2 3}$ & 0 & 520 & 0 & 0 & 0 & 0 \\
Tláhuac (9900) & 0 & 1926 & 754 & 0 & 0 & 0 & 4511 & 725 \\
Área CDMX (136840) & 1495 & $\mathbf{1 2 7 0 7 5}$ & 37248 & 36506 & 3418 & 27306 & 64039 & 48038 \\
Área conurbada (136659) & 0 & $\mathbf{1 1 6 3 3 3}$ & 9080 & 0 & 2010 & 20559 & 6162 & 8704 \\
Área total (273 499) & 0 & $\mathbf{2 4 3 4 0 7}$ & 46328 & 36506 & 5428 & 47865 & 70202 & 56742 \\
ND-MP & 0 & 15 & 11 & 8 & 6 & 9 & 14 & 13 \\
\hline
\end{tabular}

Cr 280 y Pb 400 de acuerdo con las Norma Oficial Mexicana; Cr 64, Cu 63, Ni 50, Pb 140 y Zn 200 de acuerdo con las normas canadienses; ND-MP: número de delegaciones que presentan metales pesados; en negritas las superficies contaminadas en más del 80\%; CDMX: Ciudad de México. 
$93 \%$ de la superficie muestreada (Fig. 3, Cuadro IV). Se observa una probable influencia de los vientos dominantes con dirección noreste-suroeste.

En el Estado de México, el total del AC que rebasa esta misma situación es de 116333 ha, correspondiente al $85 \%$ de su superficie. El total del área contaminada es de 243407 ha, correspondiente al $89 \%$ del área muestreada (Fig. 3, Cuadro IV).

La alta probabilidad de rebasar el LMP del Cr en la mayor parte de la CDMX y AC se debe a la gran diversidad de fuentes industriales en el norte de la ciudad y otras pequeñas fuentes dispersas por toda la zona metropolitana.

$\mathrm{El} \mathrm{Cr}$ ingresa al ambiente en las formas $\mathrm{Cr}$ III y Cr VI, proveniente del sistema de frenado de vehículos, de la quema de carbón de plantas eléctricas y petróleo, la producción y soldadura de acero inoxidable, la manufactura y uso de productos químicos, el curtido de cueros, la industria textil, colorantes, galvanoplastia y disposición de residuos industriales de Cr (ATSDR 2010).

Aun cuando se reporta que el Cr es cancerígeno, su regulación en el PU sólo es necesaria en trabajadores expuestos a grandes cantidades de este elemento y por tiempo prolongado, ya que se encuentra por toda el área de estudio y de manera constante constituye un riesgo para la salud humana (ATSDR 2010).

En la CDMX, a través del tiempo se han observado los siguientes cambios en las concentraciones de $\mathrm{Cr}$ en el PU de suelos: $121 \mathrm{mg} / \mathrm{kg}$ (Morton-Bermea et al. 2009), $135 \mathrm{mg} / \mathrm{kg}$ (Rodríguez-Salazar et al. 2011), 84 mg/kg (Ihl et al., 2015) y $126 \mathrm{mg} / \mathrm{kg}$ en el polvo de este estudio.

\section{Cobre}

La presencia de $\mathrm{Cu}$ con mayores probabilidades de contaminación tomando en cuenta un LMP de $63 \mathrm{mg} / \mathrm{kg}$ según las normas canadienses (CCME

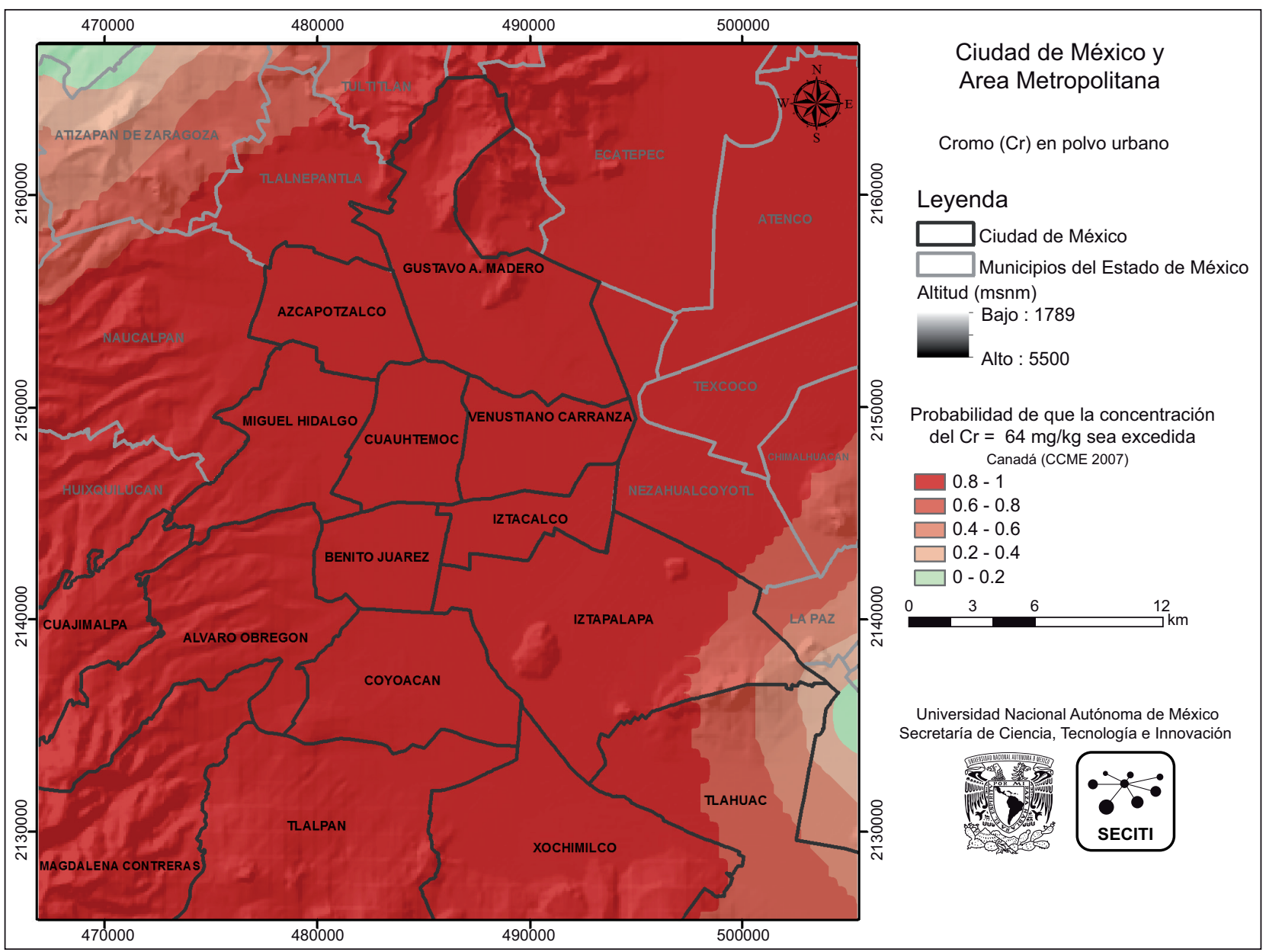

Fig 3. Áreas contaminadas con Cr en polvos urbanos de la CDMX con el LMP de $64 \mathrm{mg} / \mathrm{kg}$ establecido por las normas canadienses (CCME 2007) 
2007) abarca la totalidad de las delegaciones Azcapotzalco, Cuauhtémoc, Venustiano Carranza, Benito Juárez e Iztacalco (Fig. 4). El Cu también se encuentra en los siguientes sitios, pero de manera parcial: en el norte, en la delegación Gustavo A. Madero y el AC de Tlalnepantla y Tultitlán, Estado de México. En el este, en Netzahualcóyotl, Estado de México. En el sureste, en las delegaciones Iztapalapa y Tláhuac. En el sur, en Coyoacán. En el suroeste, en Álvaro Obregón. En el oeste, en Miguel Hidalgo y el AC de Naucalpan, Estado de México (Fig. 4).

El total del área de la CDMX contaminada por $\mathrm{Cu}$ es de 37248 ha y corresponde al $27 \%$ de su superficie; el área total contaminada conurbada con el Estado de México abarca 9080 ha, correspondiente al $6.6 \%$. El área total contaminada es de 46328 ha (33.6\%) del área muestreada (Cuadro IV).

$\mathrm{El} \mathrm{Cu}$ y sus compuestos provienen de plantas industriales, galvanoplastia, lodos de tratamiento de aguas, agua residual doméstica, combustión de desperdicios y derivados del petróleo, sistemas de frenado de vehículos de motor, hornos de ladrillos, plantas de cemento y fuentes naturales como suelos, volcanes, vegetación en descomposición e incendios forestales (ATSDR 2010).

$\mathrm{El} \mathrm{Cu}$ es esencial para mantener buena salud; sin embargo, la exposición a dosis altas o prolongadas puede ser perjudicial. El $\mathrm{Cu}$ no ha sido clasificado como carcinogénico en seres humanos porque no hay estudios al respecto, ni está regulado ambientalmente en México (ATSDR 2010).

En este estudio, el $\mathrm{Cu}$ rebasa los LMP establecidos por las normas canadienses para suelos, pero no las estadounidenses. En México no están regulados los suelos y polvos, lo cual es necesario porque se encontraron valores en PU hasta $100 \%$ mayores que en roca madre $(33.2 \mathrm{mg} / \mathrm{kg}$ ) (Morton-Bermea et al. 2009).

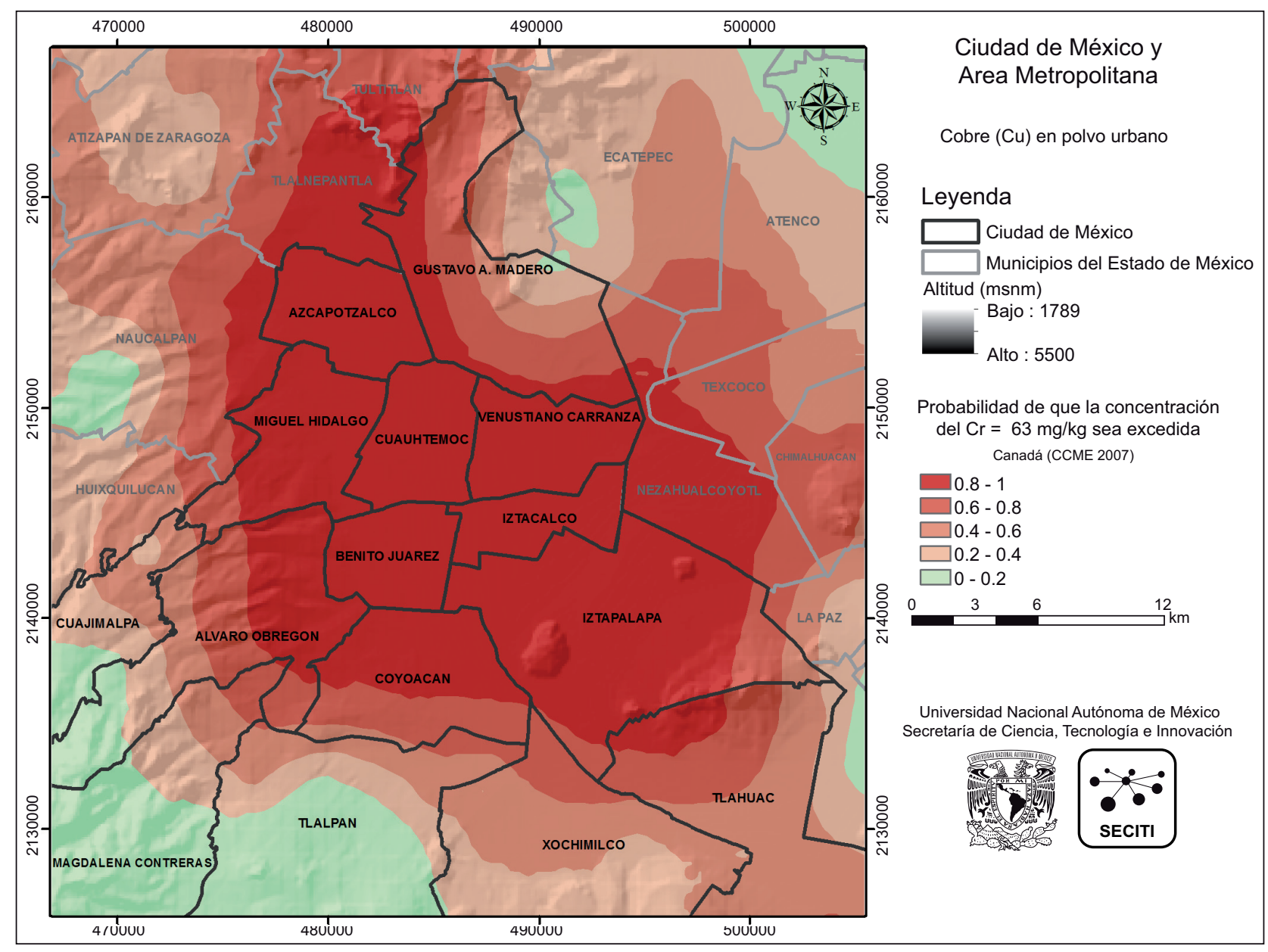

Fig 4. Áreas contaminadas con $\mathrm{Cu}$ en polvos urbanos de la CDMX con el LMP de $63 \mathrm{mg} / \mathrm{kg}$ establecido por las normas canadienses (CCME 2007) 
A través del tiempo, en la CDMX las concentraciones de $\mathrm{Cu}$ en suelos han presentado un incremento: $61 \mathrm{mg} / \mathrm{kg}$ (Morton-Bermea et al. 2009), $93 \mathrm{mg} / \mathrm{kg}$ (Rodríguez-Salazar et al. 2011), $92 \mathrm{mg} / \mathrm{kg}$ (Ihl et al. 2015) y $97 \mathrm{mg} / \mathrm{kg}$ en el polvo urbano de este estudio.

\section{Níquel}

Las áreas donde las mayores probabilidades de contaminación por Ni con el LMP de $50 \mathrm{mg} / \mathrm{kg}$ establecido por las normas canadienses (CCME 2007) se distribuyen de manera parcial en las delegaciones Gustavo A. Madero, Venustiano Carranza y Cuauhtémoc, ubicadas en la parte central de la CDMX, así como en el suroeste, en las delegaciones Cuajimalpa, Tlalpan, Álvaro Obregón y Coyoacán, y de forma total en la delegación Magdalena Contreras (Fig. 5).

En el AC con el Estado de México no hay zonas que rebasen el LMP (Fig. 5). El total del área contaminada abarca 36506 ha y corresponde al $17 \%$ del área de la CDMX y $13 \%$ del área muestreada (Cuadro IV). Al igual que en el Cr, se observa posible influencia de los vientos del noreste-suroeste.

El Ni es liberado a la atmósfera por industrias que fabrican aleaciones y otros compuestos, y como desechos en aguas residuales, plantas de energía que queman petróleo o carbón, y por incineradores de basura (ATDSR 2010).

Una gran cantidad de Ni liberada al ambiente termina en el suelo o en sedimentos, donde se adhiere fuertemente a partículas que contienen Fe y Mn. El Ni se encuentra de forma natural en la corteza terrestre, en los suelos y es liberado por volcanes (ATDSR 2010).

El Ni se adhiere fuertemente a las partículas de polvo y de tierra. Sólo las condiciones ácidas favorecen su movilización en el suelo; no obstante, es

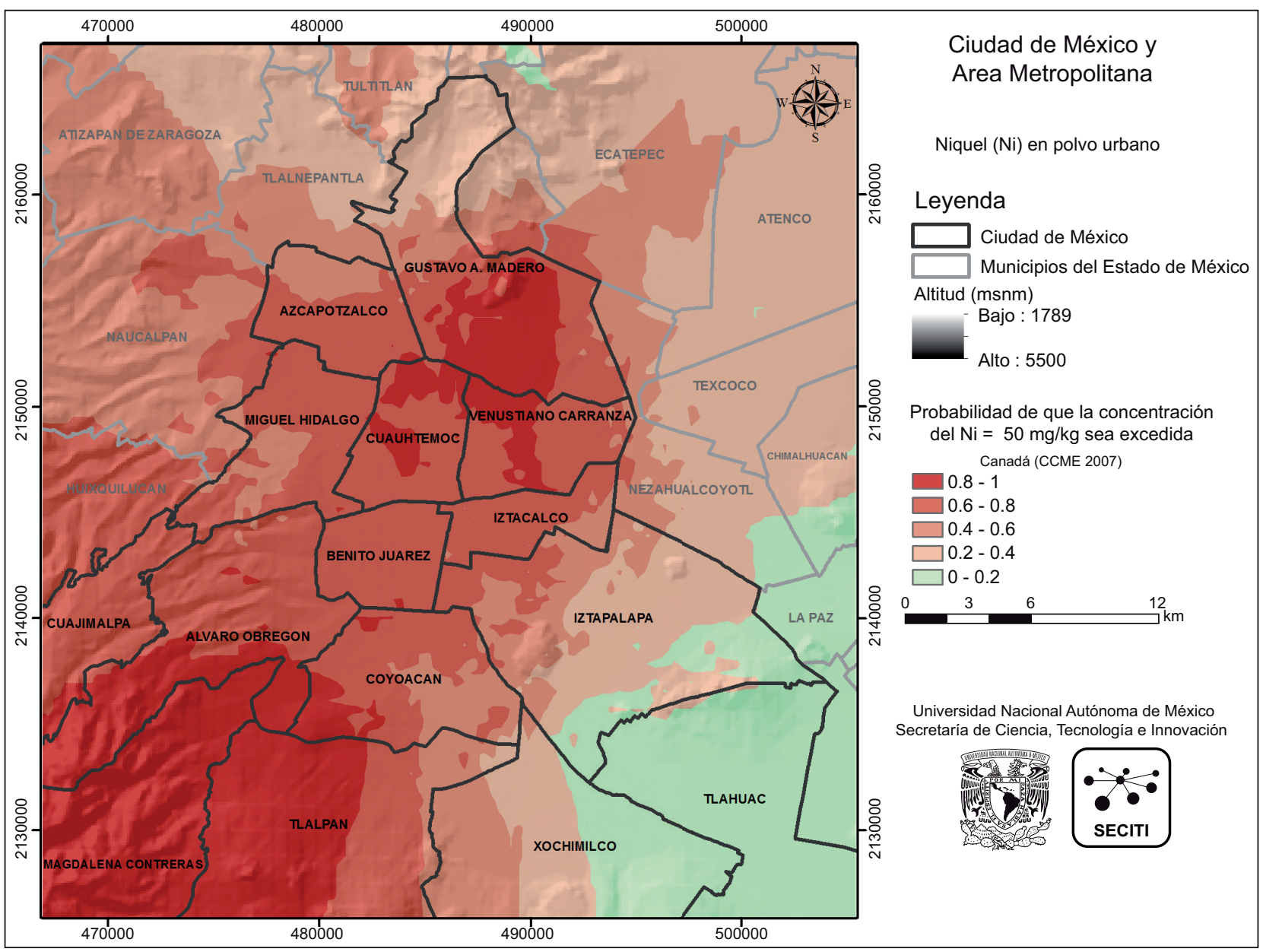

Fig 5. Áreas contaminadas con $\mathrm{Ni}$ en polvos urbanos de la CDMX con el LMP de $50 \mathrm{mg} / \mathrm{kg}$ establecido por las normas canadienses (CCME 2007) 
considerado carcinogénico cuando se encuentra en altas concentraciones, como en las plantas de procesamiento del Ni.

En este estudio, las concentraciones de Ni rebasaron los LMP establecidos por las normas canadienses para suelos, pero no las mexicanas ni las estadounidenses. Sin embargo, los polvos urbanos pueden ser más dañinos porque pueden ser inhalados.

En la CDMX existe un ligero enriquecimiento del $\mathrm{Ni}$ en el suelo a través del tiempo, considerando los valores promedio de sus concentraciones: $45 \mathrm{mg} / \mathrm{kg}$ (Morton-Bermea et al. 2009), 49 mg/kg (RodríguezSalazar et al. 2011), $45 \mathrm{mg} / \mathrm{kg}$ (Ihl et al. 2015) y 51 $\mathrm{mg} / \mathrm{kg}$ en este estudio.

Los valores del $\mathrm{Ni}$ en roca madre $(163 \mathrm{mg} / \mathrm{kg})$ (Morton-Bermea et al. 2009) rebasan los LMP de las normas canadienses, pero no los de la norma mexicana debido a que el umbral es de $1600 \mathrm{mg} / \mathrm{kg}$.

\section{Plomo}

Las probabilidades de contaminación por $\mathrm{Pb}$ con el LMP de $140 \mathrm{mg} / \mathrm{kg}$ establecido por las normas canadienses (CCME 2007) se distribuyen en la mayor parte de la ciudad, desde el noroeste hacia el sureste, en la totalidad de las delegaciones Azcapotzalco y Cuauhtémoc, y Tlalnepantla, Estado de México (Fig. 6).

De manera parcial, el $\mathrm{Pb}$ se distribuye de la siguiente manera: en el norte, en Tultitlán y Tlalnepantla, Estado de México; en el noroeste, en Atizapán de Zaragoza, y en el oeste en Naucalpan y Huixquilucan. Además, en las delegaciones céntricas como Gustavo A. Madero, Miguel Hidalgo, Venustiano Carranza, Iztacalco y Benito Juárez, y en el sur en Álvaro Obregón e Iztapalapa (Fig. 6).

El Pb abarca 27306 ha y corresponde al $20 \%$ de la superficie total de la CDMX. El AC con el Estado de México que rebasa esa misma situación es de 20

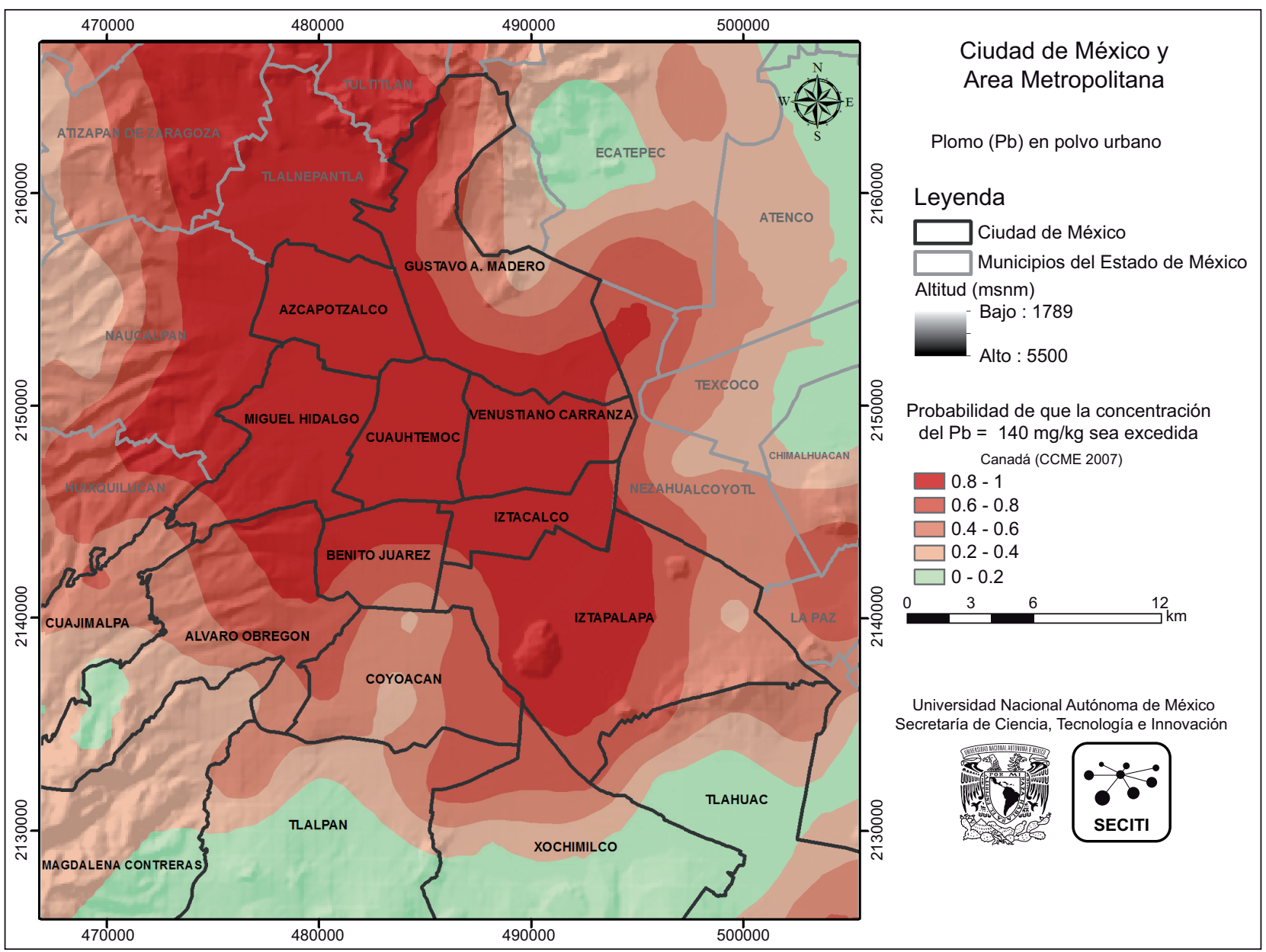

Fig 6. Áreas contaminadas con $\mathrm{Pb}$ en polvos urbanos de la CDMX con el LMP de $140 \mathrm{mg} / \mathrm{kg}$ establecido por las normas canadienses (CCME 2007) 
559 ha y corresponde al $15 \%$ del total. El total del área contaminada es de 47865 ha (17.5\%) del área muestreada (Cuadro IV).

Las áreas con mayores probabilidades de contaminación por $\mathrm{Pb}$ con el LMP de $400 \mathrm{mg} / \mathrm{kg}$ (SEMARNAT 2007) se distribuyen de manera parcial a partir del noroeste, en Atizapán de Zaragoza y Naucalpan, Estado de México. En el centro, se ubican en las delegaciones Azcapotzalco, Miguel Hidalgo, Cuauhtémoc, Benito Juárez e Iztacalco, y en el sureste en Iztapalapa (Fig. 7) El Pb abarca 3 418 ha $(2.5 \%)$ de la CDMX y 2010 ha (1.5\%) del $\mathrm{AC}$, con un total de 5428 ha (2\% del área muestreada) (Cuadro III).

En concordancia con los resultados de este trabajo, Ihl et al. (2015) utilizaron estos mismos LMP y reportaron que el $\mathrm{Pb}$ contenido en suelo rebasa los LMP en la zona centro-oeste de la CDMX. En este estudio el $\mathrm{Pb}$ en el polvo se distribuye hacia el noroeste y en una superficie mayor, ya que los contaminantes contenidos en el PU se dispersan más que los contenidos en los suelos (Bautista et al. 2011).

$\mathrm{El} \mathrm{Pb}$ puede entrar al ambiente a través de la industria del hierro y acero, quema de carbón, petróleo o desechos, manufactura de municiones y baterías, así como en la disposición de productos que contienen $\mathrm{Pb}$, soldaduras de $\mathrm{Pb}$ y uso de plaguicidas. Antes de que se prohibiera el uso de gasolina con $\mathrm{Pb}$, la mayor parte de este elemento que era liberada al ambiente provino del escape de automóviles (ATDSR 2010).

$\mathrm{E} 1 \mathrm{~Pb}$ puede permanecer adherido a partículas del suelo o sedimento en el agua durante muchos años, se encuentra disponible en el suelo en condiciones ácidas y produce cáncer cuando se incorpora al organismo humano en grandes cantidades (ATDSR 2010). En este estudio, las concentraciones de $\mathrm{Pb}$

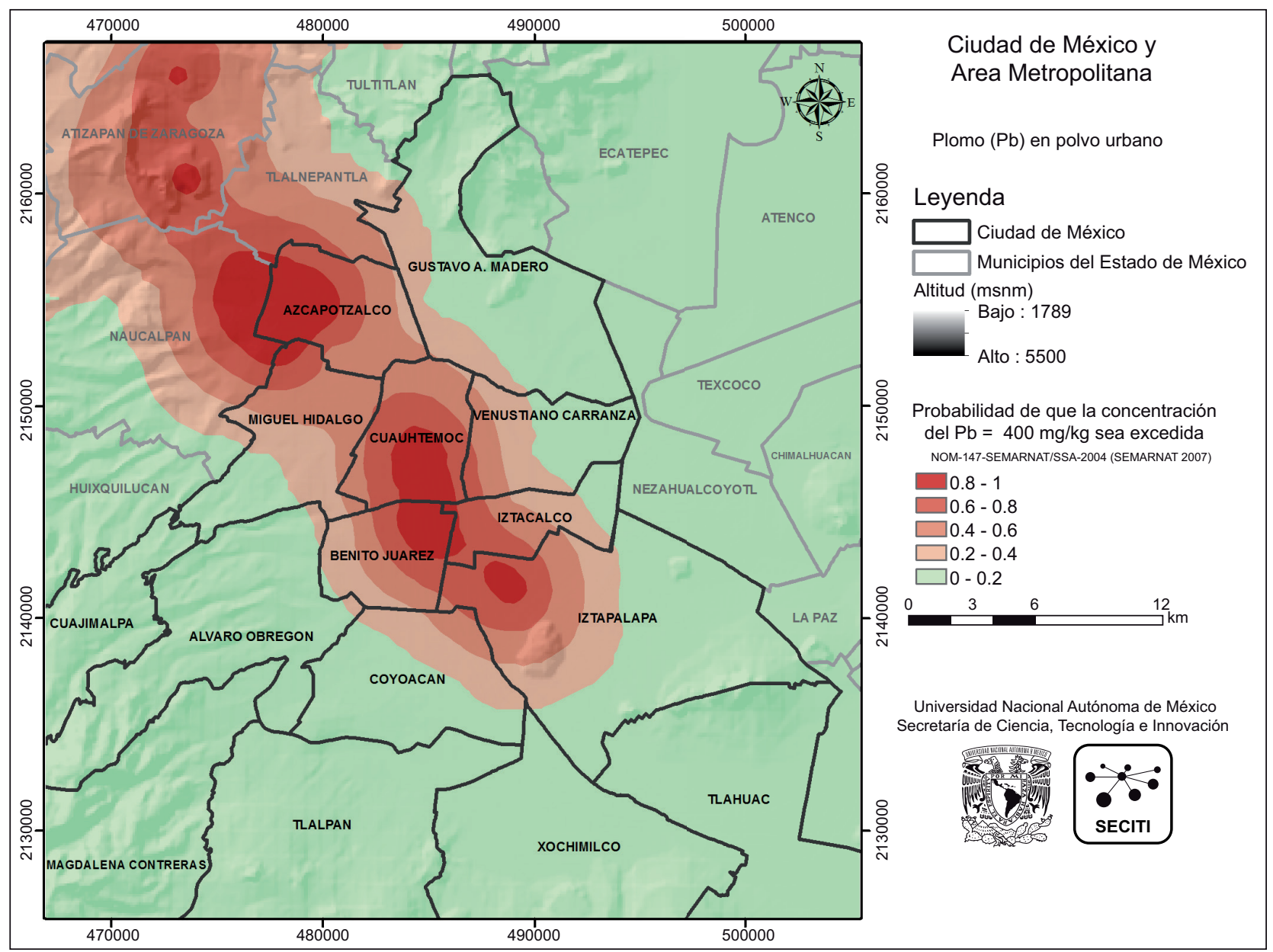

Fig 7. Áreas contaminadas con Pb en polvos urbanos de la CDMX con el LMP de $400 \mathrm{mg} / \mathrm{kg}$ establecido por las normas mexicanas (SEMARNAT 2007) 
en PU rebasan los LMP establecidos por las normas canadienses, mexicanas y estadounidenses.

A través del tiempo ha habido un aumento del $\mathrm{Pb}$ en el suelo de la CDMX considerando los promedios de sus concentraciones, que han sido hasta 22 veces mayores respecto de los valores de la roca madre (5 mg/kg) (Morton-Bermea et al. 2009): $112 \mathrm{mg} / \mathrm{kg}$ (Morton-Bermea et al. 2009), $116 \mathrm{mg} / \mathrm{kg}$ (RodríguezSalazar et al. 2011), $163 \mathrm{mg} / \mathrm{kg}$ (Ihl et al. 2015) y 206 $\mathrm{mg} / \mathrm{kg}$ en los polvos de este estudio.

\section{Vanadio}

Las zonas con mayores probabilidades de contaminación por $\mathrm{V}$ con el LMP de $78 \mathrm{mg} / \mathrm{kg}$ establecido por la norma mexicana (SEMARNAT 2007) se distribuyen del noreste hacia el sur de la ciudad (Fig. 8). El V abarca parcialmente Ecatepec y Tlalnepantla, en el Estado de México; en las delegaciones céntricas, como Gustavo A. Madero, Azcapotzalco, Miguel Hidalgo, Cuauhtémoc, Venustiano Carranza, Benito Juárez y Álvaro Obregón, y en el sur desde la Magdalena Contreras, Tlalpan, Coyoacán y Xochimilco hasta el sureste en Iztapalapa y Tláhuac (Fig. 8).

El total de la superficie que abarca el $\mathrm{V}$ en la CDMX es de 64039 ha (47\%) y 6162 ha de la zona conurbada (4.5\%), conformando un total de 70202 ha $(26 \%)$ de la superficie muestreada (Cuadro IV).

$\mathrm{Al}$ igual que ocurre con el $\mathrm{Cr}$ y el $\mathrm{Ni}$, se observa posible influencia de los vientos del noreste-suroeste, pero la diferencia es que el V se distribuye también hacia el sureste.

El V entra al ambiente mediante la actividad industrial en aleaciones de hierro y acero, colorantes para cerámica, y mediante la combustión de petróleo o carbón. De manera natural el V se encuentra en las rocas del sur de la CDMX. Se ha reportado

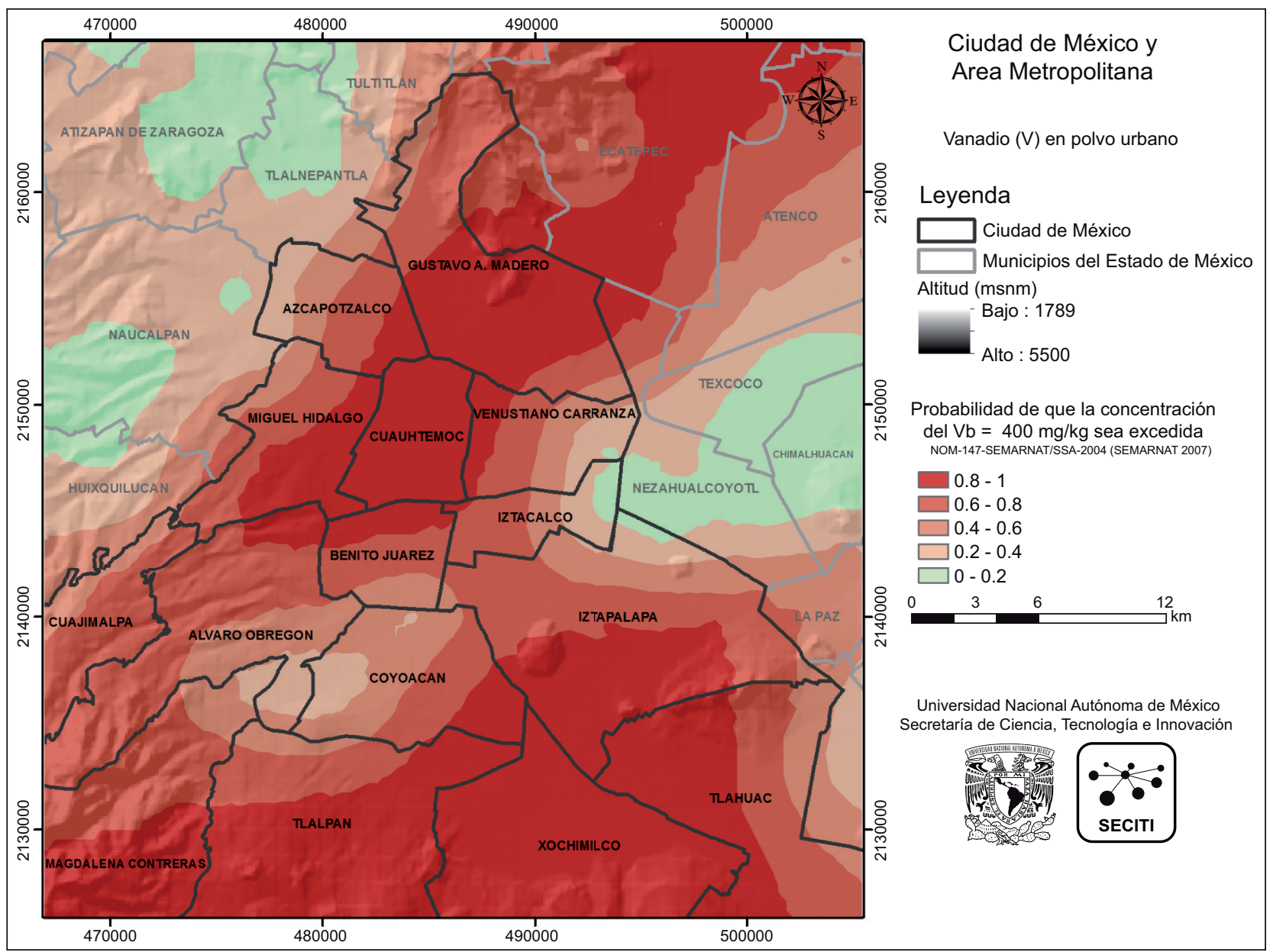

Fig 8. Áreas contaminadas con V en polvos urbanos de la CDMX con el LMP de $78 \mathrm{mg} / \mathrm{kg}$ establecido por las normas mexicanas (SEMARNAT 2007) 
como cancerígeno (Rodríguez-Mercado et al. 2006, ATDSR 2010).

\section{Zinc}

Las zonas con mayores probabilidades de contaminación por Zn con el LMP de $200 \mathrm{mg} / \mathrm{kg}$ establecido por las normas canadienses (CCME 2007) se distribuyen de manera total en las delegaciones Azcapotzalco, Cuauhtémoc, Venustiano Carranza, Benito Juárez e Iztacalco (Fig. 9). De manera parcial se sitúan en Naucalpan, Nezahualcóyotl, Tlalnepantla y Tultitlán, Estado de México, y en las delegaciones Gustavo A. Madero, Miguel Hidalgo, Álvaro Obregón, Coyoacán, Tlalpan, Xochimilco e Iztapalapa (Fig. 9).

El total del área contaminada en la CDMX es de 48038 ha, correspondiente al $35 \%$ de la superficie total, 8704 ha $(6.4 \%)$ del área conurbada y 56742 ha (21\%) del área muestreada (Cuadro IV).
El Zn se presenta de forma natural, pero las concentraciones han aumentado por las actividades industriales, la combustión de carbón y residuos, el procesamiento del acero, los catalizadores y neumáticos de autos, y los aceites de motores, fungicidas e insecticidas, aguas residuales provenientes de la industria galvánica, producción de pilas, pergaminos y pinturas (Lenntech 2017).

En este estudio se encontraron valores promedio de $\mathrm{Zn}$ de $321 \mathrm{mg} / \mathrm{kg}$, muy superiores a los contenidos de fondo y de la roca madre $(76$ y $83.7 \mathrm{mg} / \mathrm{kg}$, respectivamente) (Morton-Bermea et al. 2009).

A través del tiempo, los valores promedio del $\mathrm{Zn}$ en suelos en la CDMX han tenido las siguientes fluctuaciones: $288 \mathrm{mg} / \mathrm{kg}$ (Morton-Bermea et al. 2009), $447 \mathrm{mg} / \mathrm{kg}$ (Rodríguez-Salazar et al. 2011), $287 \mathrm{mg} / \mathrm{kg}$ (Ihl et al., 2015) y $321 \mathrm{mg} / \mathrm{kg}$ en el polvo urbano de este estudio.

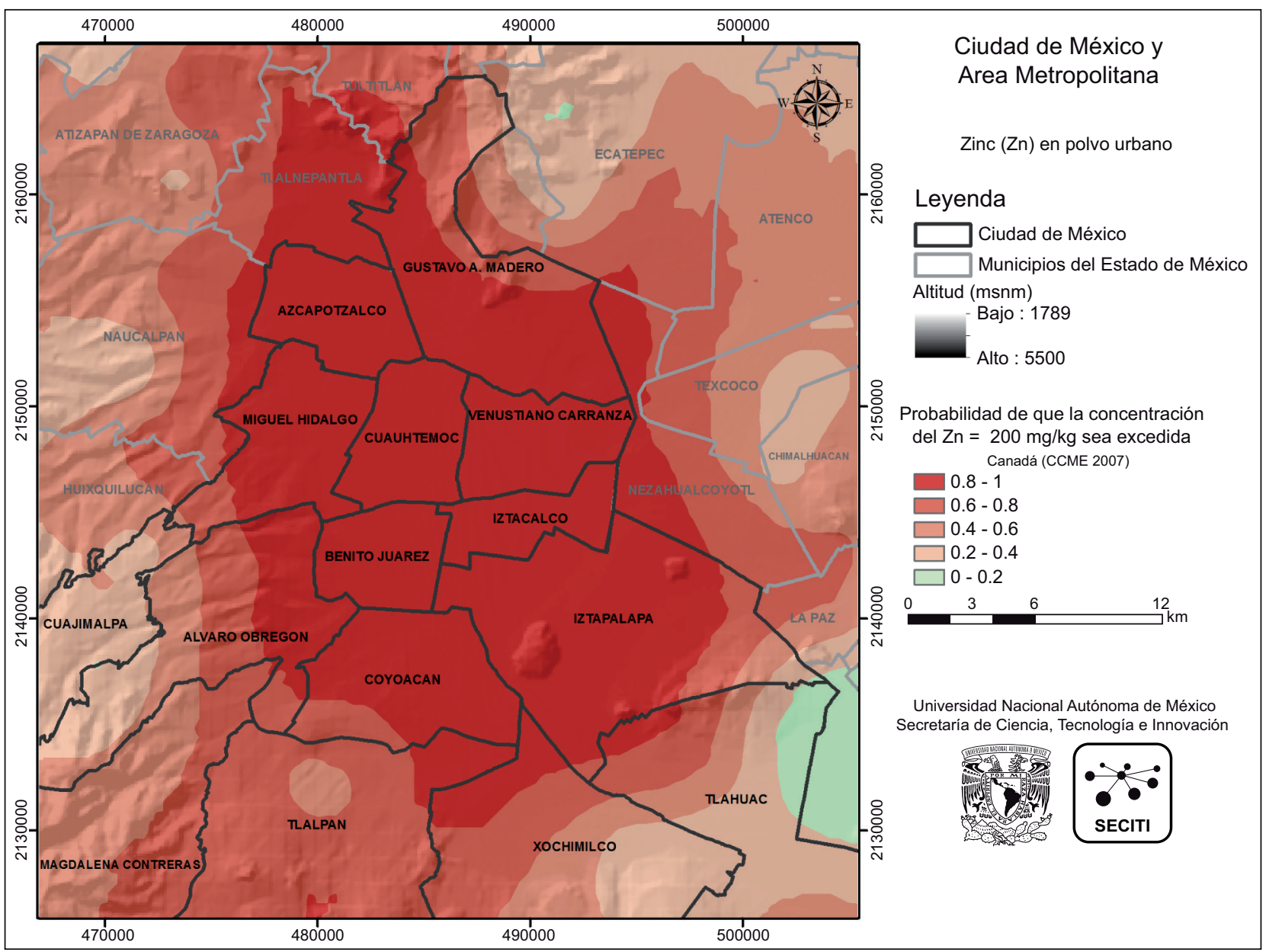

Fig 9. Áreas contaminadas con Zn en polvos urbanos de la CDMX con el LMP de $200 \mathrm{mg} / \mathrm{kg}$ establecido por las normas canadienses (CCME 2007) 


\section{Integración espacial}

El mapa de integración de las superficies contaminadas con MP de acuerdo con las normas mexicanas para $\mathrm{Cr}(280 \mathrm{mg} / \mathrm{kg})$ y $\mathrm{Pb}(400 \mathrm{mg} / \mathrm{kg})$ muestra la superficie de sobreposición del $\mathrm{Cu}, \mathrm{Ni}$, $\mathrm{Pb}, \mathrm{V}$ y Zn (Fig. 10). Abarca un área de 11678 ha y corresponde al $4 \%$ de la superficie muestreada (Cuadro V). Se ubica en las delegaciones Azcapotzalco, Cuauhtémoc, Gustavo A. Madero, Venustiano Carranza e Iztacalco.

La mayor superficie de sobreposición está definida por la asociación entre el Cu y Zn. Aabarca 39849 ha y se ubica en las delegaciones del norte y centro en dirección sureste (Fig. 10, Cuadro V).
El mapa de integración de las superficies contaminadas con MP de acuerdo con las normas mexicanas para el V $(78 \mathrm{mg} / \mathrm{kg})$ y las normas canadienses para los demás metales muestra una superficie de sobreposición del $\mathrm{Cr}, \mathrm{Cu}, \mathrm{Ni}, \mathrm{Pb}, \mathrm{V}$ y $\mathrm{Zn}$ ubicada en las delegaciones Azcapotzalco, Cuauhtémoc, Gustavo A. Madero, Venustiano Carranza e Iztacalco (Fig. 11). Abarca un área de 11706 ha que corresponde al $4 \%$ de la superficie total del área de muestreo (Cuadro VI).

Cortés et al. (2017) reportaron la confluencia de estos mismos metales ( $\mathrm{Cr}, \mathrm{Cu}, \mathrm{Ni}, \mathrm{Pb}, \mathrm{V}$ y $\mathrm{Zn}$ ) en el polvo urbano de Ensenada Baja California, México. Además, se presenta una asociación

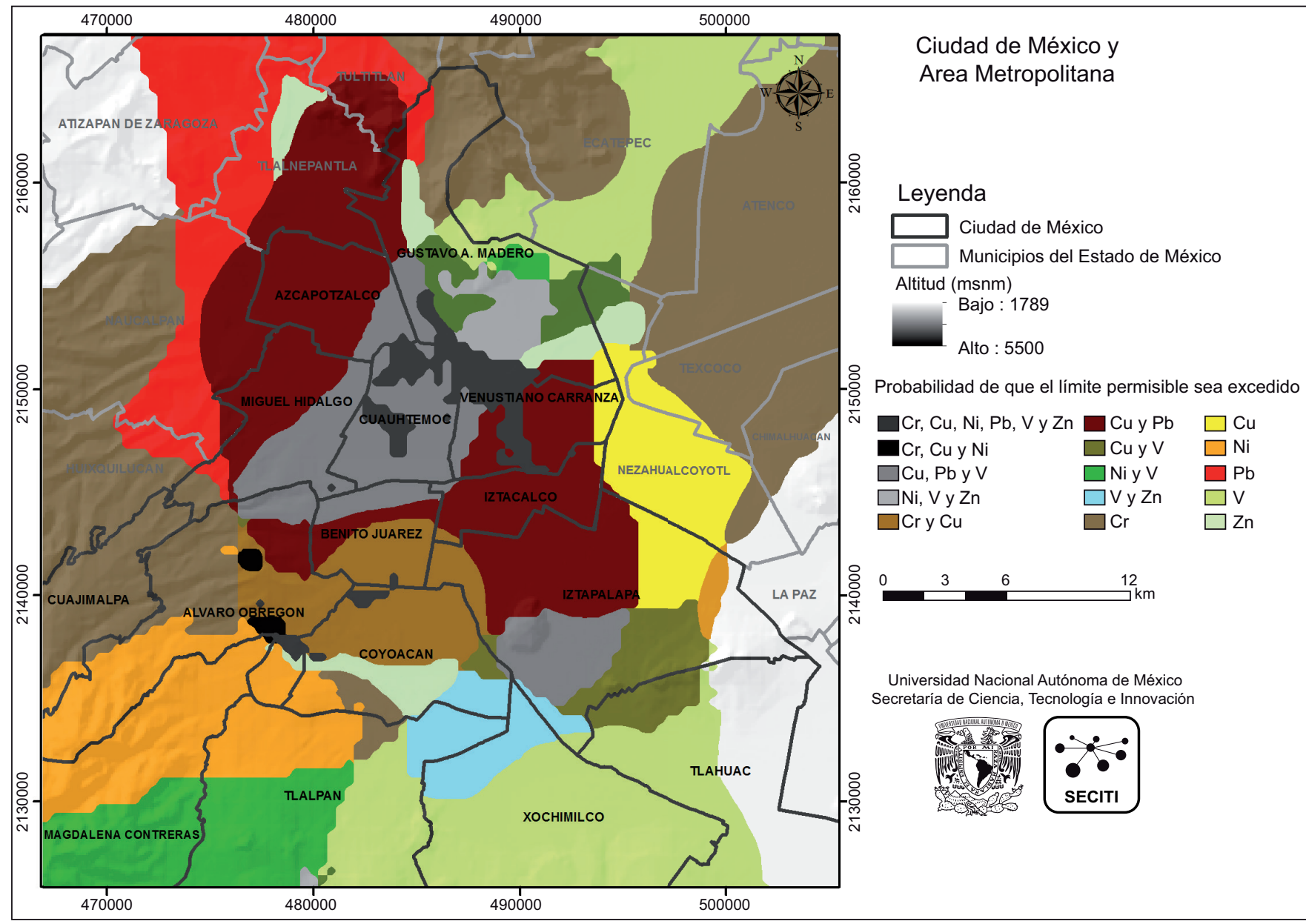

$$
\begin{array}{ll}
\mathrm{Cr}=64 \mathrm{mg} / \mathrm{kg} \text { Canadá (CCME 2007) } & \mathrm{Pb}=140 \mathrm{mg} / \mathrm{kg} \text { Canadá (CCME 2007) } \\
\mathrm{Cu}=63 \mathrm{mg} / \mathrm{kg} \text { Canadá (CCME 2007) } & \mathrm{V}=78 \mathrm{mg} / \mathrm{kg} \text { NOM-147-SEMARNAT/SSA-2004 (SEMARNAT 2007) } \\
\mathrm{Ni}=50 \mathrm{mg} / \mathrm{kg} \text { Canadá (CCME 2007) } & \mathrm{Zn}=200 \mathrm{mg} / \mathrm{kg} \text { Canadá (CCME 2007) } \\
\hline
\end{array}
$$

Fig 10. Mapa de integración de las superficies contaminadas con MP contenidos en los polvos de la CDMX de acuerdo con las normas mexicanas para $\mathrm{Cr}, \mathrm{Pb}$ y $\mathrm{V}$ y las normas canadienses para $\mathrm{Cu}, \mathrm{Ni}$ y $\mathrm{Zn}$ 
CUADRO V. SUPERFICIE CONTAMINADA POR EL CONJUNTO DE MP EN POLVOS DE LA CIUDAD DE MÉXICO DE ACUERDO CON LAS NORMAS MEXICANAS PARA CROMO, PLOMO Y VANADIO, Y NORMAS CANADIENSES PARA CROMO, COBRE, NÍQUEL, PLOMO, Y ZINC

\begin{tabular}{lcc}
\hline Metal pesado & $\begin{array}{c}\text { Superficie } \\
\text { (ha) }\end{array}$ & $\begin{array}{c}\text { Superficie } \\
(\%)\end{array}$ \\
\hline $\mathrm{Cu}, \mathrm{Ni}, \mathrm{Pb}, \mathrm{V}$ y Zn & 11678 & 4.27 \\
$\mathrm{Cu}, \mathrm{Ni}, \mathrm{Pb}$ y Zn & 4048 & 1.48 \\
$\mathrm{Cr}, \mathrm{Cu}$ y Ni & 2981 & 1.09 \\
$\mathrm{Cu}, \mathrm{V}$ y Zn & 3152 & 11.39 \\
$\mathrm{Ni}, \mathrm{V} \mathrm{y} \mathrm{Zn}$ & 8615 & 3.15 \\
$\mathrm{Cr}$ y Cu & 4786 & 1.75 \\
$\mathrm{Cu}$ y Zn & 39849 & 14.57 \\
$\mathrm{Ni} \mathrm{y} \mathrm{V}$ & 20403 & 7.46 \\
$\mathrm{~V} \mathrm{y} \mathrm{Zn}$ & 14605 & 5.34 \\
$\mathrm{Cr}$ & 5278 & 1.93 \\
$\mathrm{Cu}$ & 10174 & 3.72 \\
$\mathrm{Ni}$ & 13401 & 4.90 \\
$\mathrm{~Pb}$ & 3227 & 1.18 \\
$\mathrm{~V}$ & 35336 & 12.92 \\
$\mathrm{Zn}$ & 3665 & 1.34 \\
\hline
\end{tabular}

LMP: Cr, 280 mg/kg (SEMARNAT, 2007); Cu, 63 mg/kg (CCME, 2007); Ni, 50 mg/kg (CCME, 2007); $\mathrm{Pb}, 400$ mg/kg (SEMARNAT, 2007); V, 78 mg/kg (SEMARNAT, 2007); Zn, 200 mg/kg (CCME, 2007)

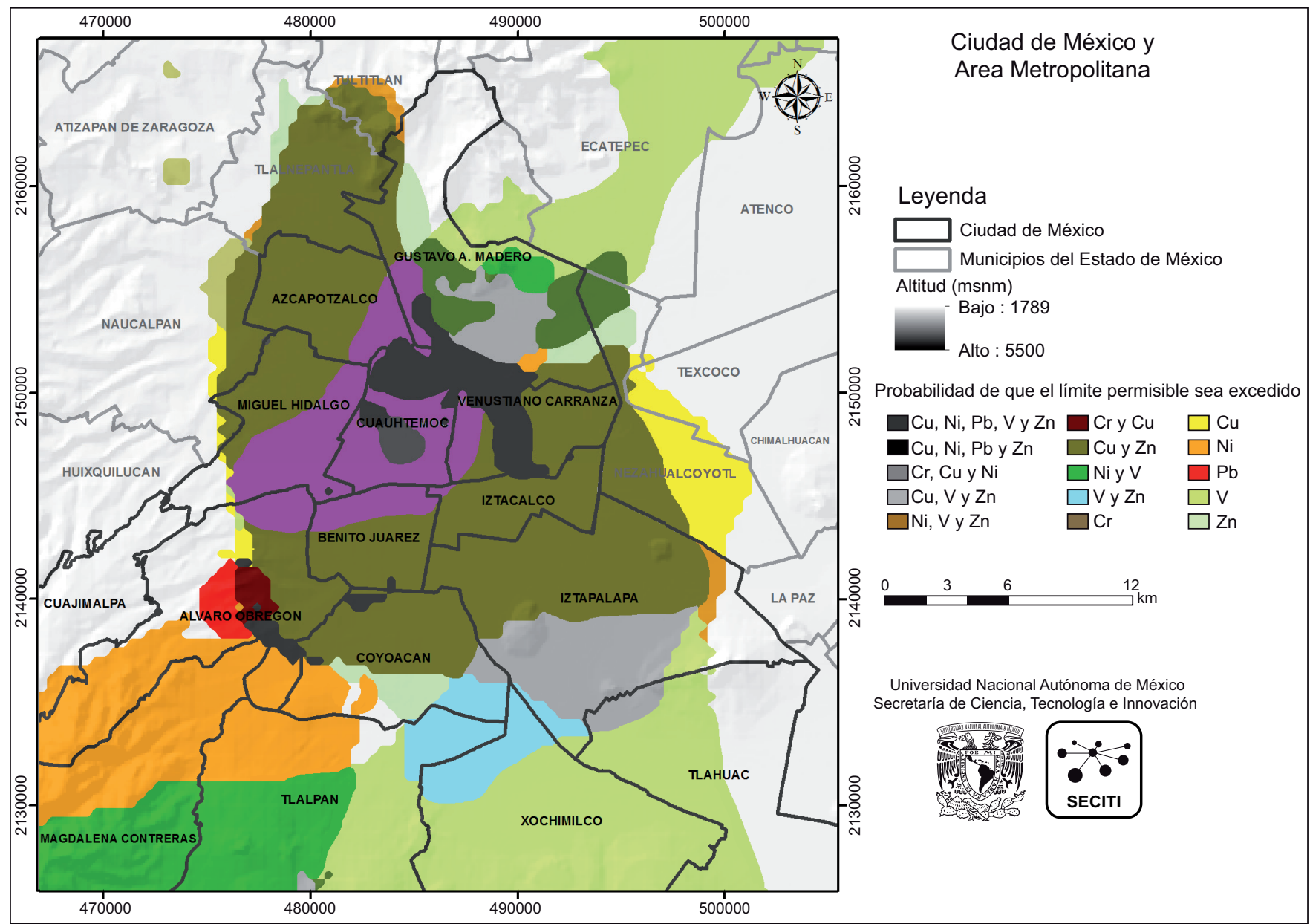

$\begin{array}{ll}\mathrm{Cr}=280 \mathrm{mg} / \mathrm{kg} \text { NOM-147-SEMARNAT/SSA-2004 (SEMARNAT 2007) } & \mathrm{Pb}=400 \mathrm{mg} / \mathrm{kg} \text { NOM-147-SEMARNAT/SSA-2004 (SEMARNAT 2007) } \\ \mathrm{Cu}=63 \mathrm{mg} / \mathrm{kg} \text { Canadá (CCME 2007) } & \mathrm{V}=78 \mathrm{mg} / \mathrm{kg} \mathrm{NOM-147-SEMARNAT/SSA-2004} \mathrm{(SEMARNAT} \mathrm{2007)} \\ \mathrm{Ni}=50 \mathrm{mg} / \mathrm{kg} \text { Canadá (CCME 2007) } & \mathrm{Zn}=200 \mathrm{mg} / \mathrm{kg} \text { Canadá (CCME 2007) }\end{array}$

Fig 11. Mapa de integración de las superficies contaminadas con MP contenidos en los polvos de la CDMX de acuerdo con las normas mexicanas para $\mathrm{V}$ y las normas canadienses para $\mathrm{Cr}, \mathrm{Cu}, \mathrm{Ni}, \mathrm{Pb}$ y $\mathrm{Zn}$ 
CUADRO VI. SUPERFICIE CONTAMINADA POR MP EN POLVOS DE LA CIUDAD DE MÉXICO DE ACUERDO CON LAS NORMAS MEXICANAS PARA EL VANADIO Y LAS NORMAS CANADIENSES PARA CROMO, COBRE, NÍQUEL, PLOMO, Y ZINC

\begin{tabular}{lcc}
\hline Metal pesado & Superficie (ha) & Superficie (\%) \\
\hline $\mathrm{Cr}, \mathrm{Cu}, \mathrm{Ni}, \mathrm{Pb}, \mathrm{V}$ y Zn & 11706 & 4.28 \\
$\mathrm{Cr}, \mathrm{Cu}$ y Ni & 1969 & 0.72 \\
$\mathrm{Cu}, \mathrm{Pb}$ y V & 26010 & 9.51 \\
$\mathrm{Ni}, \mathrm{V} \mathrm{y} \mathrm{Zn}$ & 8615 & 3.15 \\
$\mathrm{Cr}$ y Cu & 18625 & 6.81 \\
$\mathrm{Cu} \mathrm{y} \mathrm{Pb}$ & 35008 & 12.80 \\
$\mathrm{Cu} \mathrm{y} \mathrm{V}$ & 8424 & 3.08 \\
$\mathrm{Ni} \mathrm{y} \mathrm{V}$ & 11952 & 4.37 \\
$\mathrm{~V} \mathrm{y} \mathrm{Zn}$ & 8178 & 2.99 \\
$\mathrm{Cr}$ & 38481 & 14.07 \\
$\mathrm{Cu}$ & 2297 & 0.84 \\
$\mathrm{Ni}$ & 16875 & 6.17 \\
$\mathrm{~Pb}$ & 31835 & 11.64 \\
$\mathrm{~V}$ & 25435 & 9.30 \\
$\mathrm{Zn}$ & 5716 & 2.09 \\
\hline
\end{tabular}

LMP: Cr, 64 mg/kg (CCME, 2007); $\mathrm{Cu}, 63 \mathrm{mg} / \mathrm{kg}$ (CCME, 2007); Ni, 50 mg/kg (CCME, 2007); Pb, $140 \mathrm{mg} / \mathrm{kg}$ (CCME, 2007); V, $78 \mathrm{mg} / \mathrm{kg}$ (SEMARNAT, 2007); Zn, $200 \mathrm{mg} / \mathrm{kg}$ (CCME, 2007)

entre el $\mathrm{Cu}$ y $\mathrm{Pb}$ que abarca la mayor superficie de sobreposición (35 008 ha) (Cuadro VI), ubicada en las delegaciones del norte y centro en dirección sureste (Fig. 11).

En ambos mapas de integración, donde coincide el mayor número de MP, se conformaron superficies de tamaño muy semejante con una diferencia de 18 ha debido a que en el mapa diseñado con los umbrales de las normas canadienses se aumenta la superficie ocupada por el $\mathrm{Cr}$.

\section{Superficie contaminada por metales pesados por delegación}

Las delegaciones que presentan más del $80 \%$ de superficie con el mayor número de MP, que además tienen las probabilidades más altas de rebasar los LMP, son: Cuauhtémoc, con cinco metales $(\mathrm{Cr}, \mathrm{Cu}$, $\mathrm{Pb}, \mathrm{V}$ y Zn); Azcapotzalco, Iztacalco, Venustiano Carranza y Miguel Hidalgo, con cuatro metales $(\mathrm{Cr}$, $\mathrm{Cu}, \mathrm{Pb}$, y $\mathrm{Zn}$ ) (Cuadro IV).

Los MP que se presentan en el mayor número de delegaciones son: $\mathrm{Cr}$ (nivel: $64 \mathrm{mg} / \mathrm{kg}$ ) en todas las delegaciones; $\mathrm{V}$ en 14, $\mathrm{Zn}$ en $13, \mathrm{Cu}$ y $\mathrm{Ni}$ en $11, \mathrm{~Pb}$ (nivel: $140 \mathrm{mg} / \mathrm{kg}$ ) en ocho, $\mathrm{Pb}$ (nivel: $400 \mathrm{mg} / \mathrm{kg}$ ) en seis y $\mathrm{Cr}$ (nivel: $280 \mathrm{mg} / \mathrm{kg}$ ) en una (Cuadro IV).
El porcentaje total de superficie ocupada por cada MP presenta el siguiente orden: $\mathrm{Cr}$ (nivel: $64 \mathrm{mg} / \mathrm{kg}$ ) el $89 \%$, V el $26 \%$, Zn el $21 \%$, Pb (nivel: $140 \mathrm{mg} / \mathrm{kg}$ ) el $17.5 \%$, Cu el $17 \%$, Niel $13 \%$, Pb (nivel: $400 \mathrm{mg} / \mathrm{kg}$ ) el $2 \%$ y Cr (nivel: $280 \mathrm{mg} / \mathrm{kg}$ ) el $1 \%$ (Cuadro IV).

\section{Asociaciones entre metales pesados}

$\mathrm{El} \mathrm{Cu}$ y $\mathrm{Zn}$ tienen un coeficiente de correlación más alto que el resto de los elementos $(r=0.76, p<$ 0.05) (Cuadro VII). El análisis de conglomerados del $\mathrm{Cu}$ y $\mathrm{Zn}$ (Fig. 12) y su distribución espacial (Figs. 4 y 9, respectivamente) ratifican esta asociación, ya que su conformación en el espacio es muy semejante. Ambos MP ocupan el $100 \%$ de la superficie en las mismas cinco delegaciones céntricas y casi en la misma proporción.

El total de la superficie que ocupa el $\mathrm{Cu}$ es de 37248 ha y el Zn de 48038 ha. Esta diferencia de 10790 ha obedece a que el $\mathrm{Zn}$ se presenta en las delegaciones Tlalpan, y Xochimilco, mientras que el $\mathrm{Cu}$ no lo hace (Cuadro IV).

El Zn y Ni tienen un coeficiente de correlación de $0.70(\mathrm{p}<0.05)$ (Cuadro VII). El análisis de conglomerados ratifica esta asociación (Fig. 12); sin embargo, su distribución espacial no es tan semejante como entre el $\mathrm{Zn} \mathrm{y} \mathrm{Cu}$, ya que las probabilidades más altas de rebasar los LMP en el Zn se distribuyen

CUADRO VII. MATRIZ DE CORRELACIONES ENTRE LOS METALES PESADOS EN POLVOS URBANOS DE LA CIUDAD DE MÉXICO

\begin{tabular}{lccccc}
\hline & $\mathrm{Cr}$ & $\mathrm{Cu}$ & $\mathrm{Ni}$ & $\mathrm{Pb}$ & $\mathrm{V}$ \\
\hline $\mathrm{Cu}$ & 0.34 & & & & \\
$\mathrm{Ni}$ & 0.45 & & & & \\
$\mathrm{~Pb}$ & 0.22 & 0.47 & 0.24 & & \\
$\mathrm{~V}$ & 0.36 & & 0.33 & & \\
$\mathrm{Zn}$ & 0.54 & 0.76 & 0.70 & 0.48 & 0.35 \\
\hline
\end{tabular}

$\mathrm{r}=$ factor de correlación; $\mathrm{P}<0.05$

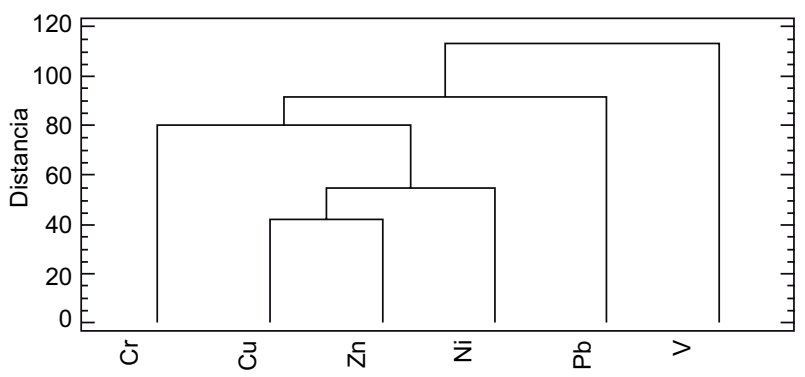

Fig. 12. Análisis de conglomerados entre los metales pesados contenidos en polvos de la CDMX 
principalmente en el centro de la ciudad y el Ni en su mayor parte en la zona montañosa del suroeste (Fig. 5 y 9).

Además de las correlaciones positivas existentes entre el $\mathrm{Zn}, \mathrm{Pb}$ y $\mathrm{Cu}(\mathrm{p}<0.05)$ (Cuadro VII), se observa también una correlación espacial tanto en los sitios donde se ubican las áreas con mayor probabilidad de rebasar los LMP (Fig. 4, 6 y 9), como en el tamaño de las superficies ocupadas: Zn, 56742 ha; $\mathrm{Pb}, 47865$ ha, y Cu, 46328 ha (Cuadro IV).

\section{CONCLUSIONES}

El análisis de la distribución espacial de cada uno de los metales pesados utilizando la interpolación KI permitió la identificación de los patrones de dispersión, así como de las zonas que rebasan los límites máximos permisibles.

El interpolador KI y el álgebra de mapas ArcGis son dos herramientas que permiten un análisis espacial integrado, con lo cual se logran mapas de gran utilidad para la instrumentación de planes de remediación de sitios contaminados en zonas urbanas.

Las delegaciones más contaminadas con metales pesados son Cuauhtémoc, Venustiano Carranza y Gustavo A. Madero. Allí se localizan las zonas que requieren medidas de remediación urgentes.

Los mapas integrados con las zonas de mayor probabilidad de rebasar los umbrales de contaminación (los LMP) para cada metal pesado permiten identificar las zonas más contaminadas considerando todos los metales pesados analizados. Las áreas señaladas en los mapas integrales deberán ser de atención prioritaria por parte de las autoridades.

\section{AGRADECIMIENTOS}

A la Secretaría de Ciencia, Tecnología e Innovación de la Ciudad de México por el apoyo financiero a través del proyecto SECITI/051/2016. Agradecemos al Consejo Nacional de Ciencia y Tecnología (CONACyT) por el apoyo financiero CB-2011-01169915.

\section{REFERENCIAS}

Aguilar Reyes B., Bautista F., Gogichaishvili A., Quintana P., Carvallo C. y Battu J. (2013). Rock-magnetic properties of topsoils and urban dust from Morelia (>800,000 inhabitants), Mexico: Implications for an- thropogenic pollution monitoring in Mexico's medium size cities. Geofis. Int. 52 (2), 121-133.

DOI: 10.19155/geofint.2013.052.2.1528

Antunes I.M.H.R. y Alburqueque M.T.D. (2013). Using indicator kriging for the evaluation of arsenic potential contamination in an abandoned mining area (Portugal). Sci. Total Environ. 442 (1), 545-552.

DOI: $10.1016 /$ j.scitotenv.2012.10.010

ATSDR (2010). Resúmenes de salud pública. Manganeso, plomo, vanadio. Agencia para Sustancias Tóxicas y el Registro de Enfermedades [en línea]. https://www. atsdr.cdc.gov/es/index.html 02/05/2016

Bautista F., Cram S. y Sommer I. (2011). Suelos. En: Técnicas de muestreo para manejadores de recursos naturales (Bautista F., Palacio J.L. y Delfín H., Eds.). Centro de Investigaciones en Geografía Ambiental, Instituto de Geografía, Universidad Nacional Autónoma de México, CDMX, México, pp. 227-258.

Beckhoff B., Kanngießer B., Langhoff N., Wedell R. y Wolff H. (2006). Handbook of practical X-ray fluorescence analysis. Springer-Verlag, Berlin Heidelberg, Alemania, 878 pp. DOI: 10.1007/978-3-540-36722-2

Burrough P.A. y McDonnell R.A. (1998). Principles of geographical information systems. Spatial information systems and geostatistics. Oxford University Press, Nueva York, EUA, 333 pp

CCME (2007). Soil quality guidelines for the protection of environmental and human health, agricultural, residential/parkland, commercial, industrial. Summary table. Canadian Council of Ministers of the Environment [en línea]. http://ceqg-rcqe.ccme.ca/en/index. html 27/04/2018

Cejudo R., Bautista F., Quintana P., Delgado-Carranza M.C., Aguilar D., Goguichaishvili A. y MoralesContreras J.J. (2015a). Correlación entre elementos potencialmente tóxicos y propiedades magnéticas en suelos de la Ciudad de México para la identificación de sitios contaminados: definición de umbrales magnéticos. Rev. Mex. Cien. Geol. 32 (1), 50-61.

Cejudo R., Goguichaishvili A., Bautista F., Delgado C., Quintana P., Aguilar D., Ramos S., Morales J., Soler A.M., Cossio-Pérez I., Hernández A. y López-Loera H. (2015b). Caracterización magnética de polvo urbano y plantas en la Zona Metropolitana del Valle de México. Latinmag. Letters 5 (5), 1-16.

Cortés J.L., Bautista F., Delgado C., Quintana P., Aguilar D., García A., Figueroa C. y Gogichaishvili A. (2017). Spatial distribution of heavy metals in urban dust from Ensenada, Baja California, Mexico. Rev. Chapingo Ser. Cien. Fores. Amb. 23 (1), 47-60. DOI: 10.5154/r.rchscfa.2016.02.005

Chen H., Lu X., Li L.Y., Gao T. y Chang Y. (2014). Metal contamination in campus dust of Xi'an, China: A study 
based on multivariate statistics and spatial distribution. Sci. Total Environ. 484, 27- 35.

DOI: $10.1016 /$ j.scitotenv.2014.03.026

Díaz-Rodríguez J. (2006). Los suelos lacustres de la ciudad de México. Revista Internacional de Desastres Naturales, Accidentes e Infraestructura Civil 62 (2), 111-129.

ESRI (2004). ArcGIS 9. Getting started with ArcGIS software. Environmental Systems Research Institute, Redlands, CA, EUA.

FIMEVIC (2012). Diagnóstico de la movilidad de las personas en la Ciudad de México. Parque vehicular, predominio del transporte privado. Fideicomiso para el mejoramiento de las vías de comunicación del Distrito Federal. Fideicomiso para el Mejoramiento de las Vías de Comunicación en el Distrito Federal [en línea]. http://www.fimevic.df.gob.mx/ problemas/1diagnostico.htm\#parque 15/02/2016

García-Rico L., Meza-Figueroa D., Gandolfi A.J., Del Río-Salas R., Romero F.M. y Meza-Montenegro M. (2016). Dust-metal sources in an urbanized arid zone: implications for health-risk assessments. Arch. Environ. Con. Tox. 70 (3), 522-533.

DOI: $10.1007 / \mathrm{s} 00244-015-0229-5$

Goovaerts P. (1999). Geostatistics in soil science: State of-the-art and perspectives. Geoderma 89, (1-2), 1-45. DOI: 10.1016/S0016-7061(98)00078-0

Guvenç N., Alagha O. y Tuncel G. (2003). Investigation of soil multi-element composition in Antalya, Turkey. Environ. Int. 29 (5), 631-40.

DOI: $10.1016 / \mathrm{S} 0160-4120(03) 00046-1$

Guzmán-Morales J., Morton-Berma O., Hernández-Álvarez E., Rodríguez-Salazar M.T., García-Arreola M.E. y Tapia-Cruz V. (2011). Assessment of atmospheric metal pollution in the urban area of Mexico City, using Ficus benjamina as biomonitor. Bull. Environ. Contam. Toxicol. 86 (5), 495-500.

DOI: $10.1007 / \mathrm{s} 00128-011-0252-9$

Ihl T., Bautista F., Cejudo R., Delgado C., Quintana P., Aguilar D. y Gogichaishvili A. (2015). Concentration of toxic elements in topsoils of the metropolitan area of Mexico City: a spatial analysis using Ordinary kriging and Indicator kriging. Rev. Int. Contam. Ambien. 31 (1), 47-62.

Jarup L. (2003). Hazards of heavy metal contamination. Brit. Med. Bull. 68, 168-182.

DOI: $10.1093 / \mathrm{bmb} / \mathrm{ldg} 032$

Juang K.W., Chen Y.S. y Lee D.Y. (2004). Using sequential indicator simulation to assess the uncertainty of delineating heavy-metal contaminated soils. Environ. Pollut. 127, 229-238.

DOI: 10.1016/j.envpol.2003.07.001

Khodeir M., Shamy M., Alghamdi M., Zhong M., Sun H., Costa M., Chen L. y Maciejcczyk P. (2012). Source apportionment and elemental composition of PM2.5 and PM10 in Jeddah City, Saudi Arabia. Atmos. Pollut. Res. 3 (3), 331-40.

DOI: 10.5094/ APR.2012.037

Lenntech B.V. (2017). Water treatment solutions. Tabla periódica. Elementos [en línea]. http://www.lenntech. es/periodica/elementos/fe.htm 14/01/2017

Liu Q., Liu Y., Yin J., Zhang M. y Zhang T. (2014). Chemical characteristics and source apportionment of PM10 during Asian dust storm and non-dust storm days in Beijing. Atmos. Environ. 91, 85-94.

DOI: 10.1016/j.atmosenv.2014.03.057

Lozano R. y Bernal J.P. (2005). Characterization of a new set of eight geochemical reference materials for XRF major and trace element analysis. Rev. Mex. Cien. Geol. 22 (3), 329-344.

Luo X.S., Yu S., Zhu Y.G. y Li X.D. (2012). Trace metal contamination in urban soils of China. Sci. Total Environ. 421-422, 17-31. DOI: 10.1016/j.scitotenv.2011.04.020

Molina L.T., Madronich S., Gaffney J.S., Apel E., de Foy B., Fast J., Ferrare R., Herndon S., Jiménez J.L., Lamb B., Osornio-Vargas A.R., Russell P., Schauer J.J., Stevens P.S., Volkamer R. y Zavala M. (2010). An overview of the MILAGRO 2006 Campaign: Mexico City emissions and their transport and transformation. Atmos. Chem. Phys. 10, 8697-8760.

DOI: $10.5194 / \mathrm{acp}-10-8697-2010$

Morton-Bermea O., Hernández-Álvarez E., GonzálezHernández G., Romero F. Lozano R. y BeramendiOrosco L.E. (2009). Assessment of heavy metal pollution in urban topsoils from the metropolitan area of Mexico City. J. Geochem. Explor. 101 (3), 218-224. DOI: $10.1016 /$ j.gexplo.2008.07.002

Paltridge N.G., Palmer L.J., Milham P.J., Guild G.E. y Stangoulis J.C.R. (2012). Energy-dispersive X-ray fluorescence analysis of zinc and iron concentration in rice and pearl millet grain. Plant Soil 361 (1-2), 251-260. DOI: 10.1007/s11104-011-1104-4

Robertson G.P. (2008). GS+: Geostatistics for the environmental sciences, v. 9. Gamma Design Software, Plainwell, Michigan, EUA, 172 pp.

Rodríguez-Mercado J.J. y Altamirano-Lozano M.A. (2006). Vanadio: contaminación, metabolismo y genotoxicidad. Rev Int. Contam. Ambie. 22 (4), 173-189.

Rodríguez-Salazar M.T., Morton-Bermea O., Hernández Álvarez E., Lozano R. y Tapia-Cruz V. (2011). The study of metal contamination in urban topsoils of Mexico City using GIS. Environ. Earth Sc. 62 (5), 899-905. DOI: $10.1007 / \mathrm{s} 12665-010-0584-5$

Sabath E. y Robles-Osorio M.L. (2012). Medio ambiente y riñón: nefrotoxicidad por metales pesados. Nefrología 32 (3), 279-286.

DOI: 10.3265/Nefrologia.pre2012.Jan.10928 
Sánchez-Duque A., Bautista F., Goguichaishvili A., Cejudo R., Reyes-López J.A., Solís-Domínguez F.A. y Morales-Contreras J.J. (2015). Evaluación de la contaminación ambiental a partir del aumento magnético en polvos urbanos. Caso de estudio en la ciudad de Mexicali, México. Rev. Mex. Cien. Geol. 32 (3), 501-513.

SEMARNAT (2007). Norma Oficial Mexicana NOM147-SEMARNAT/SSA1-2004. Que establece criterios para determinar las concentraciones de remediación de suelos contaminados por arsénico, bario, berilio, cadmio, cromo hexavalente, mercurio, níquel, plata, plomo, selenio, talio y/o vanadio. Secretaría de Medio Ambiente y Recursos Naturales. Diario Oficial de la Federación, 2 de marzo.

USEPA (2004). A preliminary remediation goals table. Region 9: The Pacific southwest. United States Environmental Protection Agency (en línea). http://www. epa.gov/region09/waste/sfund/prg/files/04prgtable. pdf $22 / 08 / 2015$
Vallejo M., Jáuregui-Renaud K., Hermosillo A.G., Márquez M.F. y Cárdenas M. (2003). Efectos de la contaminación atmosférica en la salud y su importancia en la Ciudad de México. Gac. Med. Mex. 139 (1), 57-63.

Webster R. y Oliver M.A. (1990). Statistical methods in soil and land resource survey. Oxford University Press, Nueva York, EUA, 316 pp.

Wei B. y Yang L. (2010). A review of heavy metal contaminations in urban soils, urban road dusts and agricultural soils from China. Microchem. J. 94 (2), 99-107. DOI: $10.1016 /$ j.microc.2009.09.014

WHO (2014). 7 million premature deaths annually linked to air pollution. World Health Organization [en línea]. http:/www.who.int/mediacentre/news/releases/2014/ air-pollution/en/ 12/04/2018

Zafra C.A., Temprano J. y Tejero M.J.M. (2011). Distribution of the concentration of heavy metals associated with the sediment particles accumulated on road surfaces. Environ. Technol. 32 (9), 997-1008.

DOI: $10.1080 / 09593330.2010 .523436$ 